\title{
Atom probe tomography field evaporation characteristics and compositional corrections of
}

\section{$\mathrm{ZrB}_{2}$}

David L. J . Engberg, Lina Tengdelius, Hans Högberg, Mattias Thuvander and Lars Hultman

The self-archived postprint version of this journal article is available at Linköping University Institutional Repository (DiVA):

http:// urn.kb.se/ resolve?urn=urn:nbn:se:liu:diva-160356

N.B.: When citing this work, cite the original publication.

Engberg, D. L. J., Tengdelius, L., Högberg, H., Thuvander, M., Hultman, L., (2019), Atom probe tomography field evaporation characteristics and compositional corrections of ZrB2, Materials Characterization, 156, 109871. https:// doi.org/ 10.1016/j.matchar.2019.109871

Original publication available at:

https:// doi.org/ 10.1016/j.matchar.2019.109871

Copyright: Elsevier

http://www.elsevier.com/

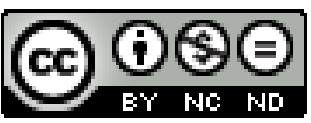




\title{
Atom Probe Tomography Field Evaporation Characteristics and Compositional Corrections of $\mathrm{ZrB}_{2}$
}

\author{
David L. J. Engberga ${ }^{*}$, Lina Tengdeliusa ${ }^{a}$, Hans Högberga, Mattias Thuvanderb, and \\ Lars Hultmana \\ a Thin Film Physics Division, Department of Physics (IFM), Linköping University, \\ SE-581 83 Linköping, Sweden \\ b Department of Physics, Chalmers University of Technology, \\ SE-412 96 Göteborg, Sweden \\ ${ }^{*}$ Corresponding author: david.engberg@liu.se
}

\section{Abstract}

The microstructure of stoichiometric $\mathrm{ZrB}_{2.0}$ and $\mathrm{B}$ over-stoichiometric $\mathrm{ZrB}_{2.5}$ thin films has been studied using atom probe tomography (APT), X-ray diffraction, and transmission electron microscopy. Both films consist of columnar $\mathrm{ZrB} 2$ grains with $\mathrm{AlB}_{2}-$ type crystal structure. The narrow stoichiometry range of $\mathrm{ZrB}_{2}$ results in the presence of separate disordered B-rich boundaries even in $\mathrm{ZrB}_{2.0}$. At higher average B content, specifically $\mathrm{ZrB}_{2.5}$, the formation of a continuous network around the sides of the $\mathrm{ZrB}_{2}$ columns is promoted. In addition, the APT field evaporation characteristics of $\mathrm{ZrB}_{2}$ and its influence on the measured local composition has been studied and compared to the average composition from elastic recoil detection analysis (ERDA). Differences in the measured average compositions of the two techniques are explained by the APT detector dead-time/space. A new pile-up pairs correction procedure based on coevaporation correlation data was thus employed here for the APT data and compared with the ${ }^{10} \mathrm{~B}$-method (the B equivalence of the ${ }^{13} \mathrm{C}$-method), as well as the combination of both methods. In $\mathrm{ZrB}_{2.0}$, all of the applied compositional correction methods were found to reduce the compositional difference when appropriate isotopic abundances were used. In $\mathrm{ZrB}_{2.5}$, the inhomogeneity of the film likely increased the local APT composition to such an extent that even conservative correction procedures overestimated the B content compared to the ERDA reference. The strengths of the pile- 
up pairs correction compared the ${ }^{10} \mathrm{~B}$ - and the combined methods are higher precision, due to it being less dependent on the accuracy of estimated isotopic abundances, and that the correction itself is not dependent on careful background correction of the mass spectrum.

\section{Keywords}

- Atom probe tomography (APT)

- Zirconium diboride $\left(\mathrm{ZrB}_{2}\right)$

- Field evaporation characteristics of borides

- Elastic recoil detection analysis (ERDA)

- Compositional correction procedures

- Transition metal diborides

\section{Introduction}

Transition metal diborides are promising materials systems with potential applications in fields such as wear-resistant coatings and electrical contacts. For wear resistance, the focus of studies has long been on nitrides and carbides, with systems of increasing complexity and commercial applications. Many of these materials have matured to a point where compounds need to be explored to further improve properties required for different kinds of cutting applications.

Ceramic boride thin films have been suggested as the next generation of wear resistant coatings [1-3]. In contrast to nitrides and carbides, which generally have large homogeneity ranges [4] and the possibility to form solid solutions with $\mathrm{N}, \mathrm{C}$, and $\mathrm{O}$ [5], borides often have narrow composition and homogeneity ranges [6,7]. Difficulties with controlling the ratio between $\mathrm{B}$ and metal components during film growth have hampered the use of boride materials systems, but recent improvements in thin film growth show great promise [8-12]. For metal cutting applications, transition metal diborides are of particular interest, as these coatings often have high hardness and good chemical stability [1]. In addition, the mutual full solubility of many diborides [13] can be advantageous for alloying.

$\mathrm{TiB}_{2}$ is the most studied transition metal diboride system $[11,14-18]$, and $\mathrm{ZrB}_{2}$ is in 
many ways similar, e.g., both have AlB2-type crystal structure with space group 191 (P6/mmm), Strukturbericht designation C32, and tend to form columnar microstructure oriented along [0001] during synthesis by vapor-phase deposition. They are often considered line compounds, as opposed to, e.g., their corresponding carbide and nitride, even though the width of the $\mathrm{TiB}_{2}$ and $\mathrm{ZrB}_{2}$ phases are $\sim 1$ at. $\%$ at temperatures below $1700^{\circ} \mathrm{C}$ in the binary alloy phase diagram [19], which incidentally is the error range for determining such lines [20]. Other $\mathrm{AlB}_{2}$-type materials systems studied include $\mathrm{NbB}_{2}$ [21], $\mathrm{CrB}_{2}$ [22,23], $\mathrm{TaB}_{2}$ [24], $\mathrm{VB}_{2}$ [25], and $\mathrm{MgB}_{2}[26,27]$. The latter has been studied in great detail because of its superconductivity up to $39 \mathrm{~K}$, while transition metal diborides are not superconducting or require lower temperatures [28,29]. Compared to many other ceramics, diborides are often decent conductors at room temperature, making them candidate systems for protection of electrical contact surfaces [30]. In addition to what applies to diborides in general, $\mathrm{ZrB}_{2}$ has a magic mismatch with GaN, making it a possible substrate for growing epitaxial GaN thin films [31-34].

Previous experiences from nitrides and carbides show that with higher film complexity, by, e.g., texture and increasing number of components, a combination of powerful analytical techniques is required to understand the relationship between growth parameters, film structure, and property enhancement. Atom probe tomography (APT) is a technique that excels in the investigation of local three-dimensional distributions of elements on the nanometer scale. The probed volume is small, but the detection threshold is very low, making it ideal also for finding trace elements and map their distribution. Paired with lattice sensitive techniques such as X-ray diffraction (XRD) and transmission electron microscopy (TEM), APT can ideally provide atomicscale characterization. However, quantitative analyzes of ceramics with APT are often influenced by their evaporation behavior in combination with the current detector technology, and borides are no exception to these circumstances [35-37]. Transition metal diboride phases have previously been studied using APT only as parts of other materials systems, such as $\mathrm{TiB}_{2}$ precipitates in steels [38] and in $\mathrm{TiN} / \mathrm{TiSi}_{2}$ layers on $\mathrm{B}$ implanted $\mathrm{Si}$ [39], or $\mathrm{HfB}_{2}$ in $\mathrm{NiAl}$ [40]. In the present in-depth APT study of transition metal diboride thin films, the $\mathrm{ZrB}_{2}$ materials system was chosen as a reference for future studies. These films have previously been investigated using XRD (including $\theta 2 \theta$-scans, pole figures, and reciprocal space maps), TEM, scanning TEM (STEM), electron energy loss spectrometry (EELS), nanoindentation, four-point probe [8], X-ray photoelectron 
spectroscopy (XPS) [41], and theoretical modeling [42]. Films for the APT analysis were primarily chosen based on the average composition and previous knowledge regarding epitaxy to the $\mathrm{Al}_{2} \mathrm{O}_{3}(001)$ substrates [8]. A high-quality, polycrystalline film, with epitaxial columnar grains and composition very close to stoichiometry, as well as a polycrystalline, non-stoichiometric (B-rich) film that is not expected to have epitaxial grains, were selected. A large deviation from stoichiometry was deemed important in order to find, clearly distinguish, and analyze the disordered grain boundaries expected from B segregation, while stoichiometric or nearly stoichiometric films are of greater interest for future applications. Even though the average compositions of the films differ, the deposition parameters were very similar. This reflects the difficulty of depositing stoichiometric transition metal diborides. Neidhardt et al. [43] found that the composition of stoichiometric to sub-stoichiometric $\mathrm{TiB}_{2}$ films is highly dependent on the pressure-distance product, pin-pointing gas-phase scattering as an important process. The compositional difference in the $\mathrm{ZrB} 2$ films of the present study may be attributed to the use of sputtering targets with different degree of erosion, since this may change the angular and energy distribution of the sputtered atoms, and thereby influence the gas-phase sputtering process. Better understanding of the film growth process requires accurate quantitative measurements of the resulting films, but determining the local distribution of elements using energy dispersive X-ray spectrometry (EDS) typically lead to underestimations of light elements, including B, while EELS of $\mathrm{ZrB}_{2}$ is difficult due to overlap of the B K edge with the $\mathrm{Zr} \mathrm{M}_{4,5}$ peak [8], thus affirming the need for development of other methods, like APT.

\section{Experimental details}

$\mathrm{ZrB}_{2}$ thin films were deposited from compound targets by a direct current magnetron sputtering (DCMS) process described previously [44,45] onto $\mathrm{Al}_{2} \mathrm{O}_{3}(0001)$ substrates for 5 or 15 min using a sputtering current of $\sim 1 \mathrm{~A}$ at a temperature of $900^{\circ} \mathrm{C}$. The substrates were held at floating potential. Prior to deposition, the substrates were degreased in 5 min sequential ultrasonic baths of trichloroethylene, acetone and isopropanol, before being blown dry with pure nitrogen.

The film composition and ${ }^{10} \mathrm{~B} /{ }^{11} \mathrm{~B}$ ratio were determined using time-of-flight energy elastic recoil detection analysis (ToF-E ERDA). The primary ion beam consisted of 36 
$\mathrm{MeV}{ }^{127} \mathrm{I}^{8+}$ and was directed onto the samples with $67.5^{\circ}$ incident angle with respect to the surface normal, while the induced recoil ions were detected at $45^{\circ}$ with respect to the incoming ion beam. The measured ERDA spectra were converted into relative atomic concentration profiles and isotope ratios using the CONTES code [46]. The conversions with the CONTES code were compared with conversions using the Potku code [47] with a close-match result. The error range of the relative atomic compositions was estimated to $5 \%$.

XRD diffractograms were recorded in a Philips PW1820 Bragg-Brentano diffractometer using a $\mathrm{Cu} \mathrm{K} \alpha \mathrm{X}$-ray source operated at $40 \mathrm{kV}$ and $40 \mathrm{~mA}$. A detector artefact at $60.90^{\circ}$ was replaced with the average signal from $60.88^{\circ}$ and $60.92^{\circ}$ in the diffractogram of $\mathrm{ZrB}_{2.5}$.

Cross-sectional TEM and plan-view STEM samples were prepared using mechanical polishing and subsequent ion milling to reach electron transparent thicknesses. TEM micrographs were recorded in an FEI Tecnai G2 TF20 UT HRTEM instrument using 200 $\mathrm{kV}$ acceleration voltage, while Z-contrast STEM images were recorded in high-angle annular dark-field mode in a double Cs-corrected FEI Titan ${ }^{3}$ microscope operated at 300 $\mathrm{kV}$ acceleration voltage.

Films for APT analyses were selected primarily based on composition, one sample very close to stoichiometric $\mathrm{ZrB}_{2}$ and one over-stoichiometric (B-rich). Specimens were prepared in a Zeiss 1540EsB CrossBeam ${ }^{\circledR}$ FIB system by employing a lift-out protocol [48]. Strips of the films, approximately $14 \times 2 \times 4 \mu \mathrm{m}^{3}$, were milled by $\mathrm{Ga}^{+}$ions at $30 \mathrm{kV}$ and pieces of the strips were welded on top of truncated Si posts by Pt deposition. The pieces were annularly milled into sharp tips and the Pt capping layer was then removed using $5 \mathrm{kV}$ acceleration voltage to reduce $\mathrm{Ga}^{+}$ion damages and implantation [49].

The APT analysis was conducted on an Imago Local Electrode Atom Probe (LEAP ${ }^{\circledR}$ ) 3000X HR system in ultra-high vacuum conditions. The maximum detection efficiency of the instrument is estimated to $\sim 37 \%$, based on the open area of the detection system. Both laser and voltage pulsing were used, although specimens run in voltage mode ruptured early on. Because of this, the results in the present study come from specimens run in laser pulsing mode, unless otherwise stated. For laser pulsing mode, the specimen base temperature was set to $25 \mathrm{~K}$, while the laser energy and frequency were $0.30 \mathrm{~nJ}$ and $200 \mathrm{kHz}$, respectively. For voltage pulsing mode, the temperature was set to $70 \mathrm{~K}$, while the pulse fraction and frequency was set to $20 \%$ and $200 \mathrm{kHz}$, respectively. The 
DC voltage applied to the specimen in both modes was continuously adjusted to maintain the probability of evaporation from a single pulse at $\sim 0.2 \%$.

APT specimens were reconstructed using the Geiser protocol [50]. Scanning electron microscopy (SEM) micrographs of the specimens were recorded for aiding the reconstruction. Approximate shank angles and radii of curvature were investigated before and after the APT analysis. Regions of lower density along the analysis direction were found in the APT analysis of the stoichiometric specimens in both laser and voltage pulsing mode, which was interpreted as the 0001 pole. The presence of the 0001 pole in the stoichiometric specimen was in itself of little use in calibrating the reconstruction, as no other poles could be identified and the field lines present close to the pole were distorted, probably due to the polycrystalline nature of the sample. However, the increased order of the ions, especially $\mathrm{Zr}$, close to the pole in the APT analysis enabled the use of the lattice spacing for adjusting the reconstruction parameters in the stoichiometric specimen against the reference spacing value $c=0.353 \mathrm{~nm}$ [51].

The volume of each atom (except $\mathrm{H}$ ) was set to $1 / 3$ of the unit cell volume. The initial radius of curvature was set to a value in the middle of the measured range and the image compression factor was adjusted to make the planes perpendicular to $c$ flat. The shank half-angle was set to a value within the measured range that also made the plane spacing consistent throughout the entire length $(z)$ of the specimen, and at the same time yielded an end radius of curvature within the measured range from SEM micrographs. Lastly, the detection efficiency was used to tune the reconstruction so that $c \approx 0.353 \mathrm{~nm}$. As there are interdependencies, the process was iterated, carefully adjusting the initial radius of curvature, shank half-angle, image compression factor, and detection efficiency in turn until the result was satisfactory, i.e. all parameters are within measured (or reasonable) ranges as well as flat, consistent $0001 \mathrm{Zr}$-planes $\sim 0.353 \mathrm{~nm}$ apart. The resulting image compression factor was 1.5 and the detection efficiency was reduced to $32 \%$.

The iteration process was used partly because the signal in spatial distribution maps [52] from the 0001 planes in the pole occasionally overlapped with the signal from alternating dense and sparse regions in hemispherical layers created in the reconstruction, which is an expected artefact in APT analyses of ceramic specimens, caused by the inability of the reconstruction algorithm to handle correlated evaporation [53]. The overlap made it impossible to reduce the signal-to-noise ratio in 1D spatial 
distribution maps, as changing parameters changed both the planes and the dense hemispherical layers. The minimum signal-to-noise ratio was found when the specimen was much wider than the measured values, because this made the dense hemispheres more planar and thus more aligned with the crystal planes of the lattice. By using 2-D spatial distribution maps, the two contributions could be somewhat separated, as the distance between regions in hemispherical layers from correlated evaporation is not consistent. Thus, the iterative process described above was applied herein.

The B over-stoichiometric specimens did not exhibit any poles and the reconstructions could thus not be calibrated in a similar way. Instead, the values of the detection efficiency and image compression factor from the reconstruction of stoichiometric $\mathrm{ZrB} 2$ were used for the B-rich specimens, while the initial specimen radius of curvature and the shank half-angle were adjusted to reflect the differences between the specimens and to make sure that the end radius of curvature was within the measured range of the concerned specimen.

The sampling parameters were investigated in accordance with the method described in [54]. The pole region of the stoichiometric $\mathrm{ZrB}_{2}$ specimens influenced the results of the sampling parameter analysis to such an extent that the parameters identified for the B-rich specimens were used for specimens of both films, namely a voxel size of $1.0 \mathrm{~nm}^{3}$ and an isotropic delocalization $3 \sigma$-value of $2.0 \mathrm{~nm}$. The increase of the delocalization in the analysis direction compared to the default setting is warranted by correlated evaporation, which increases the uncertainty of the ion positions in the analysis direction.

The sheet resistance of the films analysed by APT was measured at room temperature using a Jandel four-point probe with mA currents in forward and backward directions and at different in-plane orientations of the film. The thickness used for converting the sheet resistance to resistivity was estimated from a reference sample with known thickness as measured from cross-sectional SEM micrographs and by considering the products of the sputtering current during deposition and the deposition time of the reference sample and the investigated films. 


\section{Results}

\subsection{Elastic recoil detection analysis}

The film compositions were determined using ToF-E ERDA, in order to select films for the APT analysis and to serve as a reference for the composition measured by APT. ERDA has sensitivity for elemental ratios in the atomic mass range relevant to this study and can, in the case of $\mathrm{B}$, even distinguish the isotopes. The results from ERDA are presented in Table 1.

Stoichiometric film B over-stoichiometric film

\begin{tabular}{ccc}
\hline Zr [at. \%] & 33.0 & 28.3 \\
\hline B [at. \%] & 65.8 & 70.3 \\
\hline N [at. \%] & 0.4 & 0.4 \\
\hline $\mathbf{O}$ [at. \%] & 0.6 & 0.8 \\
\hline Ar [at. \%] & 0.2 & 0.2 \\
\hline $\mathbf{B} / \mathbf{Z r}$ & 1.99 & 2.48 \\
\hline $\mathbf{1 0}$ B $/{ }^{11} \mathbf{B}$ & 0.26 & 0.26 \\
\hline
\end{tabular}

Table 1. Composition and $\mathrm{B}$ isotope ratio of two $\mathrm{ZrB}_{2}$ films as determined by ToF-E ERDA.

Carbon is not listed in Table 1, because of partial signal overlap with B, which makes quantification uncertain, yet the distinguishable signal from $\mathrm{C}$ was insignificant compared to B.

Based on the results in Table 1, it is evident that one film is very close to stoichiometry, while the other has a significant excess of $\mathrm{B}$. The films are therefore designated $\mathrm{ZrB}_{2.0}$ for stoichiometric and $\mathrm{ZrB}_{2.5}$ for B over-stoichiometric, while $\mathrm{ZrB}_{2}$ will be used for the materials system in general. The compositions measured by ERDA are compared below with those measured by APT.

\subsection{X-ray diffraction}

XRD analyses were conducted to identify the crystal structure of both films. The XRD diffractograms of the stoichiometric and B over-stoichiometric samples are shown in Fig. 1. The signal strength from the 0001 at and 0002 peaks of the $\mathrm{ZrB} 2.0$ film is comparable to that from the $\mathrm{Al}_{2} \mathrm{O}_{3}$ substrates. Such high intensities indicate a large 
vertical coherence length, i.e., that the columns generally extend from substrate to surface. The weak contribution from 1010-oriented grains, with 1\% of the 0001-peak intensity, is an indication of defects on the $\mathrm{Al}_{2} \mathrm{O}_{3}(0001)$ surface disturbing the $\mathrm{ZrB}_{2}(0001)$ growth. $\mathrm{ZrB}_{2.5}$ has significantly lower signal strength for all the $000 \ell$ peaks, which, together with the over-stoichiometry, suggests that the B-rich film is not epitaxial. The epitaxial relationships of the columnar grains and the $\mathrm{Al}_{2} \mathrm{O}_{3}$ substrate in the stoichiometric $\mathrm{ZrB}_{2.0}$ films were reported by Tengdelius et al. [8]. It was found that the out-of-plane epitaxial relationship is $\mathrm{ZrB}_{2}(0001) \| \mathrm{Al}_{2} \mathrm{O}_{3}(0001)$, while the in-plane conditions are $\mathrm{ZrB}_{2}[10 \overline{1} 0] \| \mathrm{Al}_{2} \mathrm{O}_{3}[10 \overline{1} 0]$ and $\mathrm{ZrB}_{2}[11 \overline{2} 0] \| \mathrm{Al}_{2} \mathrm{O}_{3}[10 \overline{1} 0]$. Such films, with epitaxially-grown $\mathrm{ZrB}_{2}$ columns, can thus be used as templates for epitaxial growth of materials with suitable lattice mismatch, e.g., GaN [31-34], and applications thereof.

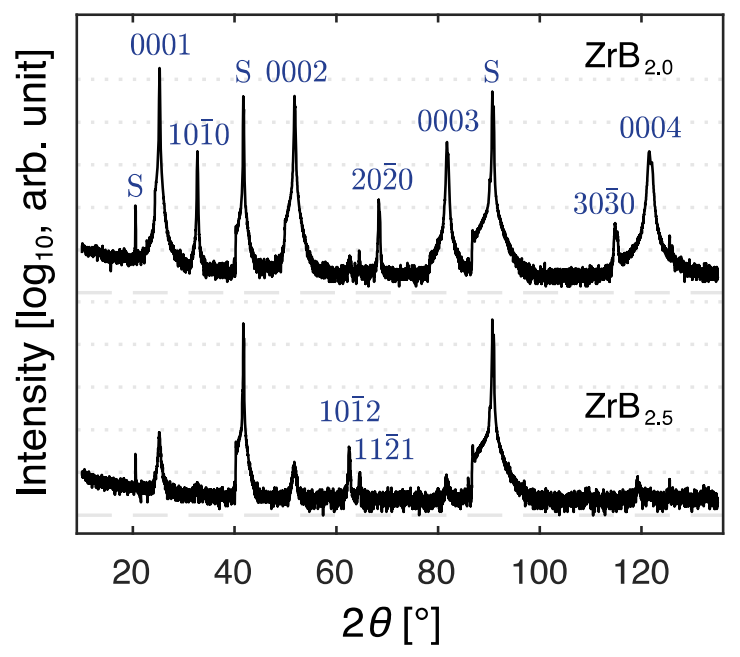

Fig. 1. XRD diffractogram of $\mathrm{ZrB}_{2.0}$ and $\mathrm{ZrB}_{2.5}$. The fine crystal quality of the stoichiometric sample is reflected in the intensity of the $000 \ell$. Substrate peaks are marked with $\mathrm{S}$.

\subsection{Transmission electron microscopy}

Typical cross-sectional TEM micrograph of the stoichiometric ZrB2.0 film in Fig. 2 a) shows $\{0001\}$-oriented columnar grains often extending from substrate to surface. The plan-view HR-STEM image in Fig. 2 b) shows that the $\mathrm{ZrB}_{2}$ film has at least two kinds of grain boundaries. Semi-coherent grain boundaries can be seen as stich-like lines separating grains while the dark regions in-between grains are interpreted as B-rich disordered grain boundaries, although clear evidence that B segregates to the grain boundary is lacking [45]. The Fourier transforms in Fig. 2 c) and d) of two neighboring 
grains in Fig. 2 b) clearly show the hexagonal pattern of the $\mathrm{AlB}_{2}$-type crystal structure. The $\sim 30^{\circ}$ rotation around the optical axis of the patterns reflects in-plane rotation of the grains, which is consistent with the pole figures in Fig. 2 of [8].

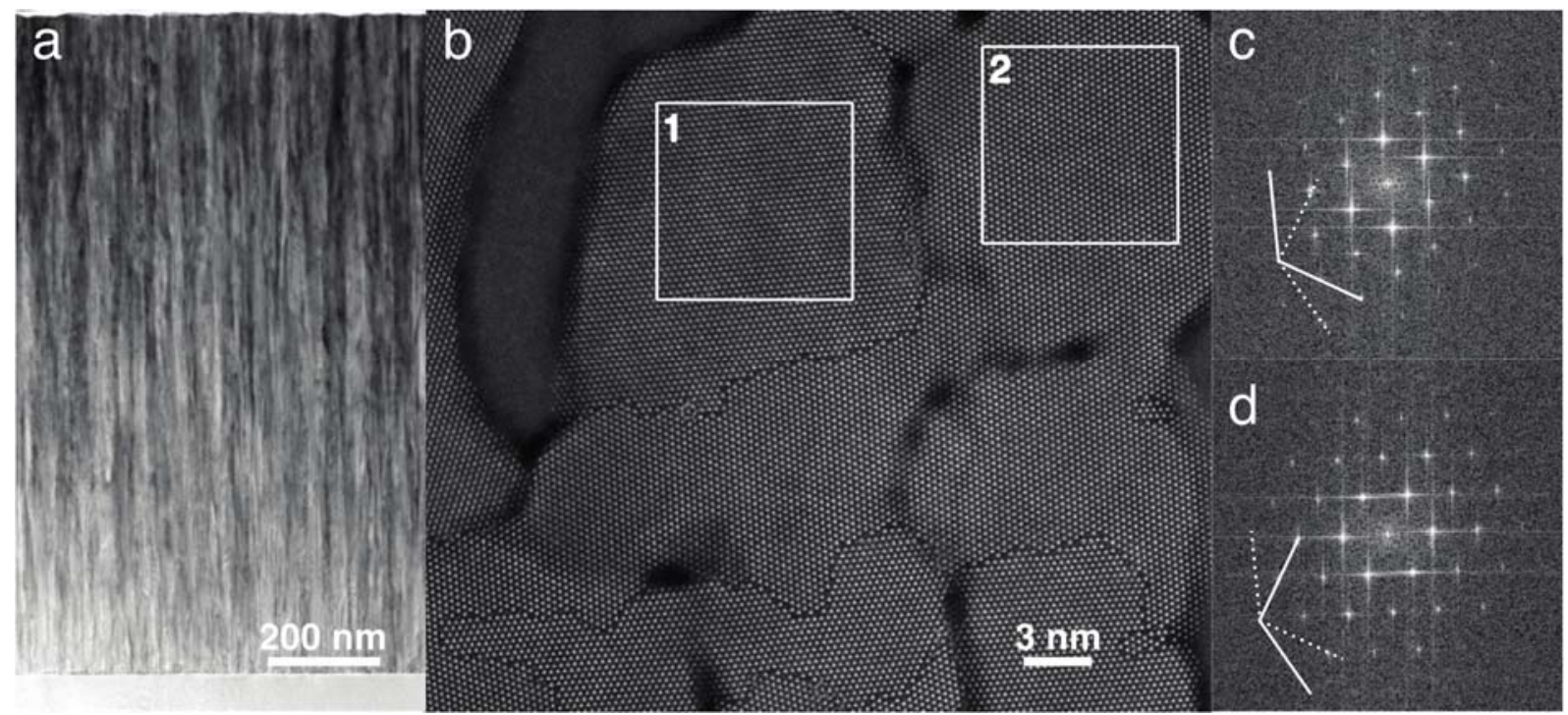

Fig. 2. Typical a) cross-sectional TEM micrograph and b) plan-view Z-contrast STEM image of $\mathrm{ZrB}_{2.0}$. The grains are columnar, often extending from substrate to surface, but with inplane lengths ranging from a few nanometers to a few tens of nanometers. Stich-like, semicoherent grain boundaries and disordered boundaries of different widths are identifiable in b). Fourier transforms from region 1 and region 2 in neighboring grains marked by squares in b) are shown in c) and d), respectively. Solid and dotted guide-lines show the $\sim 30^{\circ}$ rotation of the hexagonal patterns.

With the film average composition, crystallography, and microstructure revealed, the APT analysis will be considered next.

\subsection{Characteristics of the atom probe tomography data}

As this study concerns both the methodology of applying APT to diborides and material specific outcomes regarding $\mathrm{ZrB}_{2}$ in particular, this part focuses on the characteristics of the field evaporation and how it influences the data acquired.

\subsubsection{Mass spectra}

At first glance, interpreting the mass spectra of $\mathrm{ZrB}_{2}$ in Fig. 3 seems simple. Only one charge state of $\mathrm{Zr}$ and two of B is significant (although small $\mathrm{B}^{3+}$ peaks appear at $\sim 3.5 \mathrm{Da}$ and $\mathrm{Zr}^{2+}$ (not shown) is present in detectable numbers in the stoichiometric $\mathrm{ZrB}_{2}$ specimen only). In addition, the lack of peaks at, e.g., 10.5 Da, 21 Da and 33.67 Da in Fig. 
3 (representing, ${ }^{10} \mathrm{~B}^{11} \mathrm{~B}^{2+},{ }^{10} \mathrm{~B}^{11} \mathrm{~B}^{+}$and ${ }^{90} \mathrm{Zr}^{11} \mathrm{~B}^{3+}$, respectively) shows that the presence of molecular ions is insignificant. Lastly, no major peak overlap occurs and the mass resolution is sufficient in both specimens, although slightly better in the B overstoichiometric specimen, as can be seen when comparing the $\mathrm{Zr}^{3+}$ peaks of the two spectra in Fig. 3. Unmarked minor peaks are generally related to known trace elements in the sputtering target or gases, like Ar from the plasma in the growth chamber.

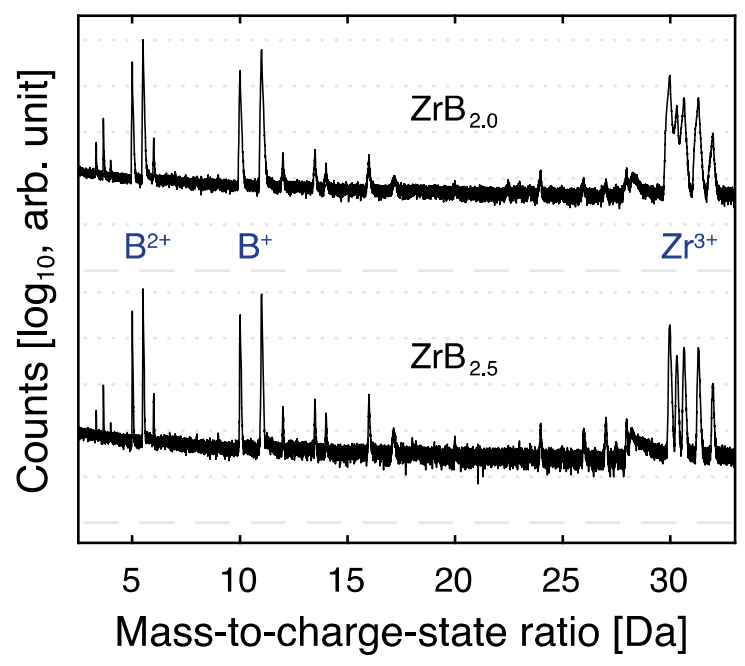

Fig. 3. APT mass spectra from $\mathrm{ZrB}_{2.0}$ and $\mathrm{ZrB}_{2.5}$ specimens with major isotope families labelled.

Closer inspection of the mass spectra reveals that the isotopic signature of B deviates slightly from what would be expected with a natural isotope abundance. The ${ }^{10} \mathrm{~B} /{ }^{11} \mathrm{~B}$ ion ratio at charge states one and two is $>0.29$ compared to $\sim 0.25$ from the natural abundance and $\sim 0.26$ from ERDA. This difference can be explained by preferential loss of ${ }^{11} \mathrm{~B}$ in multiple events during the APT detection, as discussed below.

\subsubsection{Multiple events}

The percentage of multiple events was lower than expected from previous experiences with ceramics, $29.5 \%$ for $\mathrm{ZrB}_{2.5}$, and $26.5 \%$ for $\mathrm{ZrB}_{2.0}$ for the entire analyses, while $\sim 40$ $\%$ or more is common in transition metal nitrides [54-59], carbides [60,61], carbonitrides [62], and boron carbides [63]. As can be seen in the bar chart Fig. 4, the multiple events percentage is significantly lower when considering the reconstructed volume only, i.e., after excluding parts of the analysis. Still, out of all reconstructed and ranged ions, $27.2 \%$ and $23.8 \%$ are detected in multiple events, and most of these are B 
ions, $93.1 \%$ and $90.3 \%$ in $\mathrm{ZrB}_{2.0}$ and $\mathrm{ZrB}_{2.5}$, respectively. In total, $~ 58 \%$ of all $\mathrm{B}$ ions were detected in multiple events, which is close to what Meisenkothen et al. [37] reported for the specimens included in Fig. 4; more specifically that $>60 \%$ of all ranged B ions were detected in multiples events even though the two specimen materials investigated exhibited a wide span in both concentration and multiple events percentage.

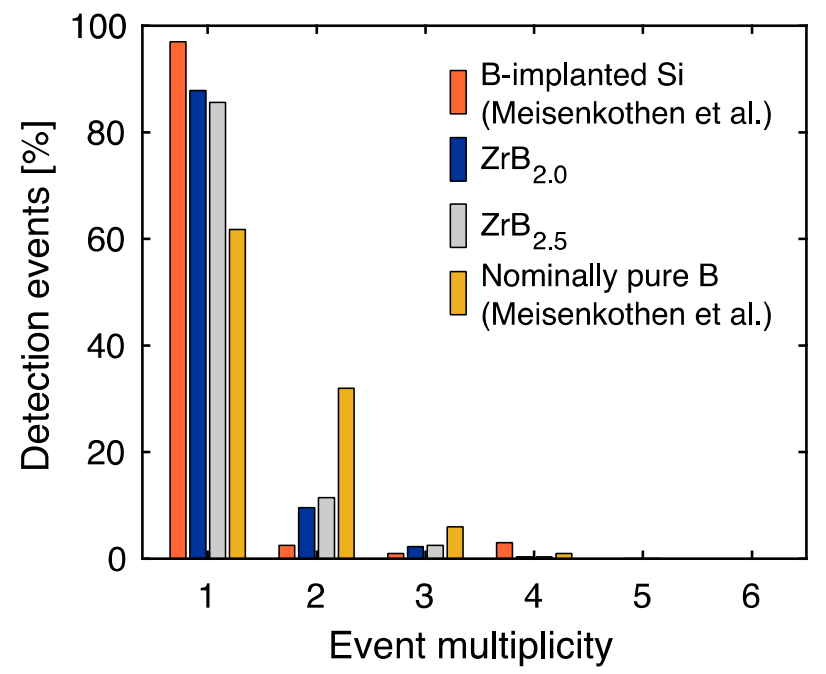

Fig. 4. Percentage of detection events as a function of number of detected ions per pulse (multiplicity) measured in the reconstructed volume, rather than the entire analysis. Approximate values from B-implanted Si and nominally pure B by Meisenkothen et al. were extracted from Fig. 11 in [37].

To further the understanding of how multiple events are influencing APT measurements, the characteristics of the field evaporation of $\mathrm{ZrB}_{2}$ is investigated next.

\subsubsection{Field evaporation characteristics}

In order to elaborate details regarding the field evaporation behavior of $\mathrm{ZrB}_{2}$, we consider the B/Zr ratio in the initial part of the APT analysis, before the equilibrium surface is formed, as well as co-evaporation correlation scatter plots [64].

The first part of an APT analysis is generally excluded, as it often contains atoms and molecules adsorbed on the specimen, damages and implanted Ga from specimen preparation. Also, the low ion energy might lead to ion-mass-dependent detection efficiencies [65], and the equilibrium surface may not yet have formed. Instructively, the first part is included in the line profile of $\mathrm{ZrB}_{2.5}$ in Fig. 5, to show the evolution of B/Zr 
before and after an equilibrium surface is formed. The B/Zr profile from a $20 \mathrm{~nm}$ wide cylinder along $z$, shown in Fig. 5, starts at 0.5 and quickly reaches 4.5 before the curve stabilizes very close to 2.4 at $z>7 \mathrm{~nm}$. The trend is similar in $\mathrm{ZrB}_{2.0}$, but with smaller fluctuations, starting from 1.7 with a maximum of 2.4 at $z=4 \mathrm{~nm}$. Since the applied voltage (and thus the ion energy) is practically constant in this short range, the drastic change in $\mathrm{B} / \mathrm{Zr}$ is not due to mass dependent detection efficiency, but rather due to preferentially retained B on the surface of the APT specimen.

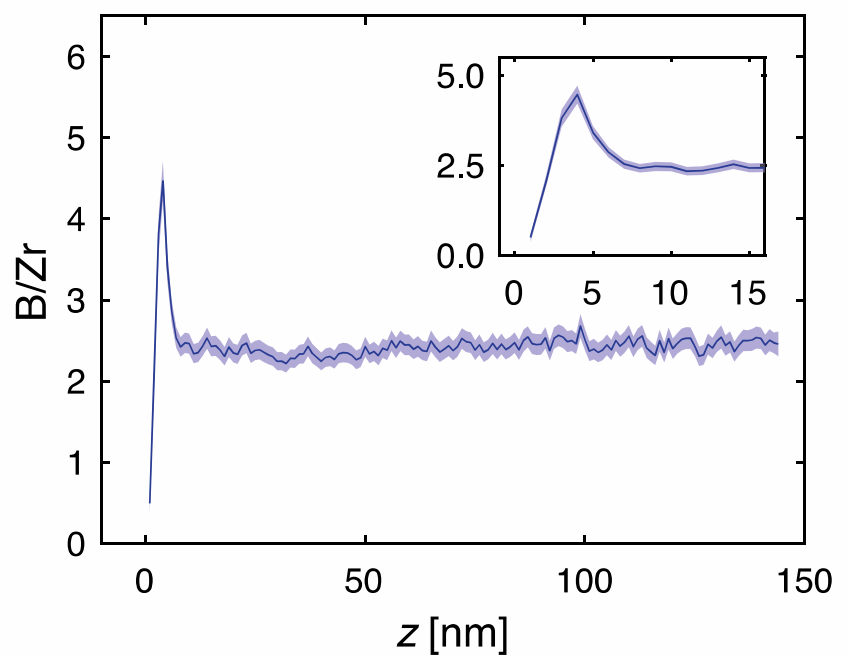

Fig. 5. Evolution of $\mathrm{B} / \mathrm{Zr}$ in $\mathrm{ZrB}_{2.5}$ along the analysis direction $(z)$ in a cylinder with $20 \mathrm{~nm}$ diameter located in the center of a reconstruction that also includes the initial part of the analysis, which is otherwise excluded. The inset highlights the peak at $z=4 \mathrm{~nm}$, located in the otherwise excluded part. The shaded area represents $2 \sigma$ error from counting statistics.

Co-evaporation correlation histograms and scatter plots are restricted to ions detected in multiple events, i.e. when more than one ion is generated in a single voltage or laser pulse and more than one ion is also detected. The spatial coordinates of the ions are ignored. Instead, the mass-to-charge-state ratio of all the second ions detected in multiple events are plotted against the mass-to-charge-state ratio of the previous (first) ion detected. A typical scatter plot for $\mathrm{ZrB}_{2}$ can be seen in Fig. 6 . 


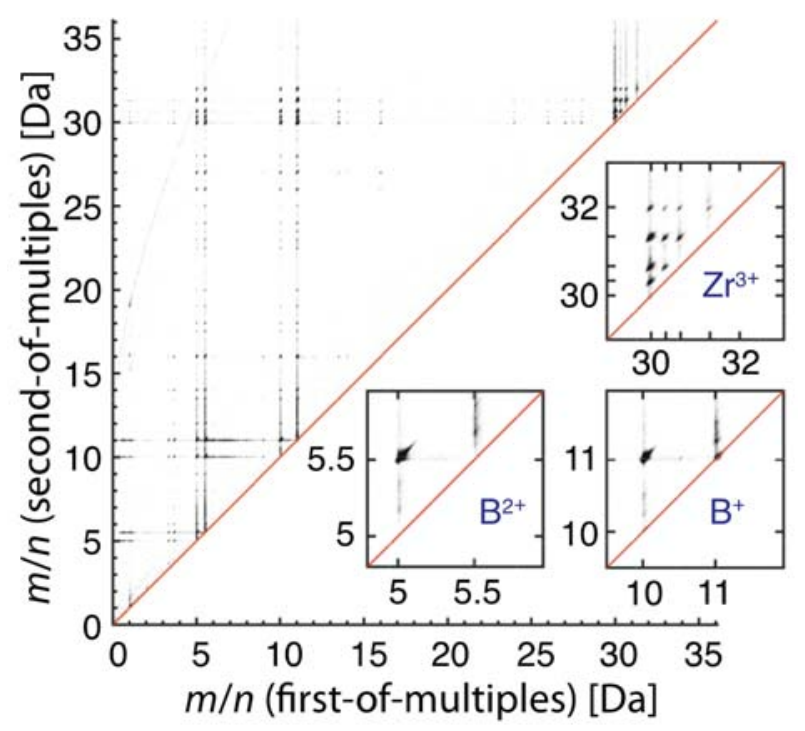

Fig. 6. Typical co-evaporation correlation scatter plot of $\mathrm{ZrB}_{2}$ with semi-transparent dots ( $5 \%$ opacity) representing ion pairs detected in multiple events. The region closest to the $x=y$ line has very few dots because it is most affected by the detector dead-time/space. Insets show enlarged regions of interest with pairs of either $\mathrm{Zr}^{3+}, \mathrm{B}^{+}$, or $\mathrm{B}^{2+}$, with tick marks at ion pair intersections from instantaneous and simultaneous field evaporation.

When the field evaporation of the two ions is instantaneous and simultaneous, a semitransparent dot is generated at the intersection of their mass-to-charge-state ratios. Horizontal lines of dots toward higher ratios are generated by pairs where the ions associated with the $x$-axis (i.e. the first ions in Fig. 6) are delayed, while vertical lines toward higher ratios represent a delay of the ions associated with the $y$-axis (i.e. the second ions in Fig. 6). When dots are found along straight lines, the timely ion is generally ranged, while delayed ions outside the mass spectrum ranges can be recovered, as long as no overlaps make the ion identity indeterminable.

The cause of curved, diagonal lines depends on their direction. Curved lines from ion intersections toward higher mass-to-charge-state ratios are created from simultaneous field evaporation that has been delayed with regards to the laser pulse. Dissociation of molecular ions can also generate curved diagonal lines in a histogram or scatter plot, but these are toward increasing ratio in one axis and decreasing in the other [64]. Dots along curved lines often have two unranged (or improperly ranged) ions [66].

In both investigated films, there are no curved lines from molecular dissociation, which is expected since the fraction of detected molecular ions is vanishingly small, although lack of dissociation tracks can also be explained by the energy compensating 
action of the reflectron $[64,67]$. The curved lines originating from the incidence point of major ions toward higher mass-to-charge-state ratios are plentiful, but generally so short that the ions are within the assigned range. This reflects the good mass resolution and short thermal tails seen in the mass spectrum in Fig. 3. Should this not be the case, a majority of ion pairs from such curved lines can often be recovered because of the characteristic time-of-flight difference $\Delta t$ [64]. By changing the $x$-axis of co-evaporation correlation histograms with unranged ion pairs to $\Delta \sqrt{m / n}$, which is proportional to $\Delta t$, $82 \%$ and $67 \%$ of all the unranged multiple ions could be recovered in $\mathrm{NiO}$ and $\mathrm{Fe}_{3} \mathrm{O}_{4}$, respectively [66].

A noticeable artefact in the scatter plots is that ion pair intersections close to the $x=$ $y$ line are clearly lacking ions. It is most evident at $5.5 \mathrm{Da}$ and $11 \mathrm{Da}$, i.e. ${ }^{11} \mathrm{~B} /{ }^{11} \mathrm{~B}$ ion pairs, but applies to all ions, as is highlighted by the insets in Fig. 6. According to the natural isotope abundance, ${ }^{11} \mathrm{~B} /{ }^{11} \mathrm{~B}$ ion pairs should be twice as common as ${ }^{10} \mathrm{~B} /{ }^{11} \mathrm{~B}$, yet the intersections of the latter have significantly more ions in the scatter plot. There is, however, no lack of ${ }^{11} \mathrm{~B} /{ }^{11} \mathrm{~B}$ ion pairs in the ${ }^{11} \mathrm{~B}^{2+} /{ }^{11} \mathrm{~B}^{+}$intersection at $5.5 \mathrm{Da} / 11 \mathrm{Da}$, and the number of ion pairs in all four intersections related to combinations of $\mathrm{B}^{2+} / \mathrm{B}^{+}$pairs does roughly reflect the natural isotope abundance. The lack of ions along the $x=y$ line can be explained by pile-up and the dead-time/space of the delay line detector. When several ions reach the detector at the same time and at close proximity, their signals will be superposed and might not be distinguishable as separate detections. An undetected ion results in a (false) single detection event (in the case of one detected ion) or a multiple detection event with too low multiplicity (in the case of two or more detected ions). In general, the greater the distance between the ions, the closer in time they may hit the detector, and vice versa, without signal superposition, and thus be detected as separate ions. However, for short (ns) differences in time, the detection efficiency also depends on the relative location of the ions on the detector, i.e., the dead-zone exhibits dynamic spatial inhomogeneities $[37,68]$. Thermally field-evaporated ion pairs, i.e., generated by the same laser pulse, generally have small kinetic energy spread [69] and if the ions also have the same mass-to-charge-state ratio, they will mainly be separated in time by the field evaporation delay. Elements with many stable isotopes of reasonably large abundances are less likely to yield multiple event ions with the same mass-tocharge-state ratio, and are thus less affected by the detector dead-time/space. With this in mind, B, with two abundant stable isotopes, is more likely lost than $\mathrm{Zr}$, with five such 
isotopes (whereof four with abundances above 10\%). Another factor influencing the total loss is how many charge states are present, as this adds variety to the possible mass-to-charge-state ratios of the ions in multiple events. The fact that B were fieldevaporated at two significant charge states $\left(\mathrm{B}^{+}, \mathrm{B}^{2+}\right)$, while $\mathrm{Zr}$ only at one $\left(\mathrm{Zr}^{3+}\right)$ in this study, could have shifted the loss ratio to the better (i.e. to have less impact on the detected composition), if not $90-93$ at. \% of the detected ions in multiple events were B, while only 5-9 at. \% were Zr ions, caused by the strong correlative field evaporation behavior of B.

Lastly, the likelihood of the element to be field evaporated as part of molecular ions and the number of different molecular ions formed could also add variety to the possible mass-to-charge-state ratios of the ions in multiple events, but this effect is marginal in the present study as very few molecular ions were detected.

To sum up, the ions most likely to be lost are those that 1) exhibit correlated field evaporation, 2) originate from the same location on the specimen, and 3) have the same mass-to-charge-state ratio. Since it is likely that the difference in evaporation behavior of $\mathrm{Zr}$ and B have shifted the composition measured by APT in this study toward lower $\mathrm{B} / \mathrm{Zr}$ ratio, a separate investigation of compositional correctional procedures is required.

\subsection{Composition correction procedures}

For materials systems exhibiting correlated field evaporation, ions lost in multiple events due to detector dead-time/space often affect the measured composition to such an extent that correction procedures are required. Larson et al. [70] have formulated a scaling factor for quantitative APT measurements of B using NIST standard reference material and a combination of APT and secondary ion mass spectrometry (SIMS), however the factor is based on Si with $<1$ at. \% B and is not applicable to specimens containing B as a major element. Thus, other such procedures have been employed here to the APT data of $\mathrm{ZrB}_{2}$. Here, an adaptation of the established ${ }^{13} \mathrm{C}$-method will be compared with the new pile-up pairs correction procedure based on the co-evaporation correlation data, as well as the combination of both methods. 


\subsection{1 ${ }^{10} \mathrm{~B}-$ method}

Thuvander et al. [60] developed a correction procedure for carbides based on the ${ }^{13} \mathrm{C}$ isotope. By using carefully background corrected mass spectral peaks of ${ }^{13} \mathrm{C}$ to quantify peaks of ${ }^{12} \mathrm{C}$ using their relative natural abundances of $\sim 1 \%$ and $\sim 99 \%$, respectively, the composition of several known carbides could be determined with better accuracy $[62,71]$. The number of detected ${ }^{13} \mathrm{C}$ ions of a certain charge state was multiplied by the percentage ratio 98.89/1.07 from the natural abundance to obtain the corrected number of ${ }^{12} \mathrm{C}$ ions of that charge state. Because of its scarcity, it is highly unlikely that ${ }^{13} \mathrm{C}$ ion pairs are generated, and the losses due to detector dead-time/space are thus minimal. The trade-off in using this procedure is increased uncertainty due to counting statistics, strong influence of the background correction, and the possibility of error due to the specimen having a different, and unknown, isotope ratio.

As mentioned in Section 3.1, the isotopic signature of B measured by APT deviates slightly from both the natural abundance and that measured by ERDA, with a ${ }^{10} \mathrm{~B} /{ }^{11} \mathrm{~B}$ isotopic ratio $>0.29$ instead of $<0.25$ (natural) and $<0.26$ (ERDA). This was explained by the co-evaporation correlation in Section 3.4.3, where much more ${ }^{11} \mathrm{~B}$ ions were lacking due to the detector dead-time/space. This commends that a similar procedure as that used for quantifying carbides could be beneficial for borides, i.e., the ${ }^{10} \mathrm{~B}$-method (in analogy with the ${ }^{13} \mathrm{C}$-method) [71,72]. In addition, the isotopic signatures of $\mathrm{C}$ and $\mathrm{B}$ have some resemblance, with one major and one minor isotope. The differences in peak ratio of the isotopic signatures (20/80 versus $99 / 1$ for ${ }^{10} \mathrm{~B} /{ }^{11} \mathrm{~B}$ and ${ }^{12} \mathrm{C} /{ }^{13} \mathrm{C}$, respectively) may, however, influence the results. As ${ }^{10} \mathrm{~B}$ is more abundant than ${ }^{13} \mathrm{C}$, the increase in uncertainty due to counting statistics is not as severe, but even with an abundance as high as $20 \%$, careful background correction of the ${ }^{10} \mathrm{~B}$ peaks is required. Moreover, the ${ }^{10} \mathrm{~B}$ method is more easily applicable than the ${ }^{13} \mathrm{C}$-method since fewer ions are required in a region of interest for reaching the detection limit of ${ }^{10} \mathrm{~B}$ compared to ${ }^{13} \mathrm{C}$, and peak overlaps due to thermal tails between the two should be less of a problem even at higher charge states, where the mass spectral peak separation is small, since ${ }^{10} \mathrm{~B}$ is lighter than ${ }^{11} \mathrm{~B}$. However, with $\sim 20 \%{ }^{10} \mathrm{~B}$, the generation of ${ }^{10} \mathrm{~B}$ ion pairs should be $\sim 400$ times more likely than ${ }^{13} \mathrm{C}$ pairs. Thus, one can expect that also some ${ }^{10} \mathrm{~B}$ is lost in multiple events. Given a known isotopic abundance, the method can either provide a conservative correction, or be further improved using an iterative process where the lost ${ }^{10} \mathrm{~B}$ ions are estimated from the number of lost ${ }^{11} \mathrm{~B}$ ions using Eq. 1 and 2 [72]. The 
corrected number of ${ }^{10} \mathrm{~B}$ ions is then used to re-calculate the corrected number of ${ }^{11} \mathrm{~B}$ ions, and so on until the number of lost ${ }^{10} \mathrm{~B}$ and ${ }^{11} \mathrm{~B}$ ions stabilize, based on the fact that the isotope ratio stipulates that loss of one ion from ${ }^{11} \mathrm{~B}$ pairs should be $\sim 16$ times more likely than from ${ }^{10} \mathrm{~B}$ pairs.

$$
\begin{gathered}
{ }^{11} \mathrm{~B}_{\text {loss }} \cdot 0.2^{2}=\left({ }^{11} \mathrm{~B}_{\text {corrected }}-{ }^{11} \mathrm{~B}_{\text {measured }}\right) \cdot 0.2^{2}={ }^{10} \mathrm{~B}_{\text {loss }} \cdot 0.8^{2} \\
{ }^{10} \mathrm{~B}_{\text {loss }}={ }^{11} \mathrm{~B}_{\text {loss }} \cdot\left(\frac{0.2}{0.8}\right)^{2}
\end{gathered}
$$

In the case of the average $\mathrm{ZrB}_{2}$ composition, errors from counting statistics is not an issue, since $B$ is the most abundant element and $\sim 10$ million ions are ranged in the reconstructions. The difference in standard deviation from counting statistics using 1.5 million ${ }^{10} \mathrm{~B}$ ions instead of 7.5 million ions of ${ }^{10} \mathrm{~B}$ and ${ }^{11} \mathrm{~B}$ is vanishingly small. However, when analyzing sub-volumes of a reconstruction, this might not be the case.

\subsubsection{Pile-up pairs correction}

Another possible correction procedure would be to use the co-evaporation correlation scatter plot to estimate the number of missing pairs due to detector dead-time/space. Similar estimations have previously been made for 1-D atom probes, where the number of detected pairs as a function of the time-of-flight difference $\Delta t$ was plotted and a curve was fitted for small $\Delta t$, where ion pairs were lacking [73]. For 3-D atom probes, the separation of the ions in space, together with a spatially and dynamically inhomogeneous detection efficiency $[37,68]$, must also be considered, which significantly complicates such a correction.

In the pile-up pairs correction, the detected number of pairs within a range close to the intersections at the $x=y$ line is increased to a value estimated using a predefined abundance (e.g. the natural or a measured) and a reference pair that, ideally, is not affected by the detector dead-time/space. Since lost pairs are most likely detected as false single ions, each pair increases the concerned ion count by one. If there are significant curved diagonal lines from mutually delayed evaporation of reference ion pairs outside the assigned range, these should first be recovered from their constant time-of-flight difference, as applied in [66], since mutually delayed field-evaporated ions with the same mass-to-charge-state ratio will also be counted as false singles.

To correct $\mathrm{ZrB} 2$ using this procedure, the ${ }^{10} \mathrm{~B}^{+} /{ }^{11} \mathrm{~B}^{+},{ }^{10} \mathrm{~B}^{2+} /{ }^{11} \mathrm{~B}^{2+},{ }^{10} \mathrm{~B}^{3+} /{ }^{11} \mathrm{~B}^{3+}$, and ${ }^{90} \mathrm{Zr}^{3+} /{ }^{94} \mathrm{Zr}^{3+}$ pairs were used as reference for ion pairs of the same charge state, and no 
recovery of mutually delayed ions was necessary. The ranges of the mass-to-chargestate ratio used to select the ion pairs in the correlation scatter plot were the same as those applied to the mass spectra in Fig. 3, which will be used to calculate the APT composition and generate composition maps.

For each charge state, the ideal percentage $P_{\alpha, \beta}$ of an ion pair with isotopes $\alpha$ and $\beta$ can be calculated from a predefined abundance using Eq. 3, where $p_{i}$ is the percentages of the isotope $i$ in the relevant abundance and $N$ is the number of stable isotopes.

$$
P_{\alpha, \beta}=\frac{p_{\alpha} \cdot p_{\beta}}{\sum_{i=1}^{N} p_{i}}=\frac{p_{\alpha} \cdot p_{\beta}}{100}
$$

It should be noted that ion pairs with different isotopes $(\alpha \neq \beta)$ can generate pairs in two varieties, $P_{\alpha, \beta}$ and $P_{\beta, \alpha}$, which is apparent when investigating pairs where the ions have different charge states in Fig. 6 . The ion pairs ${ }^{10} \mathrm{~B}^{2+} /{ }^{11} \mathrm{~B}^{+}$and ${ }^{11} \mathrm{~B}^{2+} /{ }^{10} \mathrm{~B}^{+}$at $x / y-$ coordinates $5 \mathrm{Da} / 11 \mathrm{Da}$ and $5.5 \mathrm{Da} / 10 \mathrm{Da}$ each generate a collection of pairs that (roughly) abide Eq. 3 when using percentages from the natural abundance. In the case of the reference pairs $\left({ }^{10} \mathrm{~B}^{+} /{ }^{11} \mathrm{~B}^{+},{ }^{10} \mathrm{~B}^{2+} /{ }^{11} \mathrm{~B}^{2+},{ }^{10} \mathrm{~B}^{3+} /{ }^{11} \mathrm{~B}^{3+}\right.$, and $\left.{ }^{90} \mathrm{Zr}^{3+} /{ }^{94} \mathrm{Zr}^{3+}\right)$ the two varieties coincide to one point, ideally with two times the number of pairs, because the lighter ion, ${ }^{10} \mathrm{~B}$ or ${ }^{90} \mathrm{Zr}$ in this materials system, will (almost) always be detected first when the charge state is the same.

The estimated number of an ion pair $n_{\alpha, \alpha}^{\prime}$ can then be found by multiplying the detected number of reference ions $n_{\alpha, \beta}$ of the same charge state by the percentage ratio according to Eq. 4 .

$$
n_{\alpha, \alpha}^{\prime}=n_{\alpha, \beta} \cdot \frac{p_{\alpha} \cdot p_{\alpha}}{2 \cdot p_{\alpha} \cdot p_{\beta}}
$$

Even though the lack of ion pairs in the correlation scatter plot suggests that two ions should be added for each pair, this is not the case, since it is most likely that the multiple evaporation event resulted in a false single detection event, i.e., one of the ions have already been detected. To further improve the correction, the total number of reference pairs from all multiplicities (first and third, first and fourth, etc., up to the highest measured multiplicity) must also be included in the same manner before calculating the estimated number of ion pairs, to account for multiples with too low multiplicity. Ideally, and with an abundance close to natural, ${ }^{11} \mathrm{~B} /{ }^{11} \mathrm{~B}$ pairs should be about twice as numerous as the ${ }^{10} \mathrm{~B} /{ }^{11} \mathrm{~B}$ reference pairs of the same charge state, while the number of 
${ }^{10} \mathrm{~B} /{ }^{10} \mathrm{~B}$ pairs should be about $1 / 8$ of the number of reference pairs. As previously mentioned, this is clearly not the case in Fig. 6. Since ions that are delayed beyond the specified ranges will either not be counted or not recovered completely, and that it is possible for reference pairs to also be affected by the detector dead-time/space, but to a lesser extent than pairs at the $x=y$ line, the pile-up pairs correction can be considered a conservative correction procedure.

\subsubsection{Combined correction}

Lastly, the two previous methods are combined. The pile-up pairs correction is first used to estimate the number of ${ }^{10} \mathrm{~B}$ ions lost. These, together with the detected ${ }^{10} \mathrm{~B}$ ions, are then used as the input in the ${ }^{10} \mathrm{~B}$-method. This compensates for the loss of ${ }^{10} \mathrm{~B}$-ions in multiple events, instead of using an iterative loss estimation process. This corrects for the detector dead-time/space while maintaining the benefit of better counting statistics for ${ }^{10} \mathrm{~B}$ compared to ${ }^{13} \mathrm{C}$. The combined method will thus make such isotope $\left({ }^{13} \mathrm{C}\right.$-like) methods available for elements with isotopic signatures dissimilar to that of $\mathrm{C}$ and $\mathrm{B}$ (but have at least two isotopes). Careful background correction is necessary for accurate results using this correction procedure.

\subsection{Composition}

Before applying compositional corrections, we investigate the elemental distribution of $\mathrm{B}$ and Zr using APT composition, density, and multiple event ions percentage maps in Fig. 7. To better reveal the 3-D distribution of elements, videos of $\mathrm{B} / \mathrm{Zr}$ in $\mathrm{ZrB} 2.0$ and $\mathrm{ZrB} 2.5$ along each direction $(x, y, z)$ is included as supplementary material. Since compositional corrections provide a specimen-wide factor, and this primarily regards the distribution of the elements, the composition maps of Fig. 7 and supplementary videos have not been corrected. The resulting correction would change the color scales toward slightly higher B/Zr ratio, total density, and multiple event ions percentage, since most of the lost ions are B and all ions lost are considered as multiple events ions. 

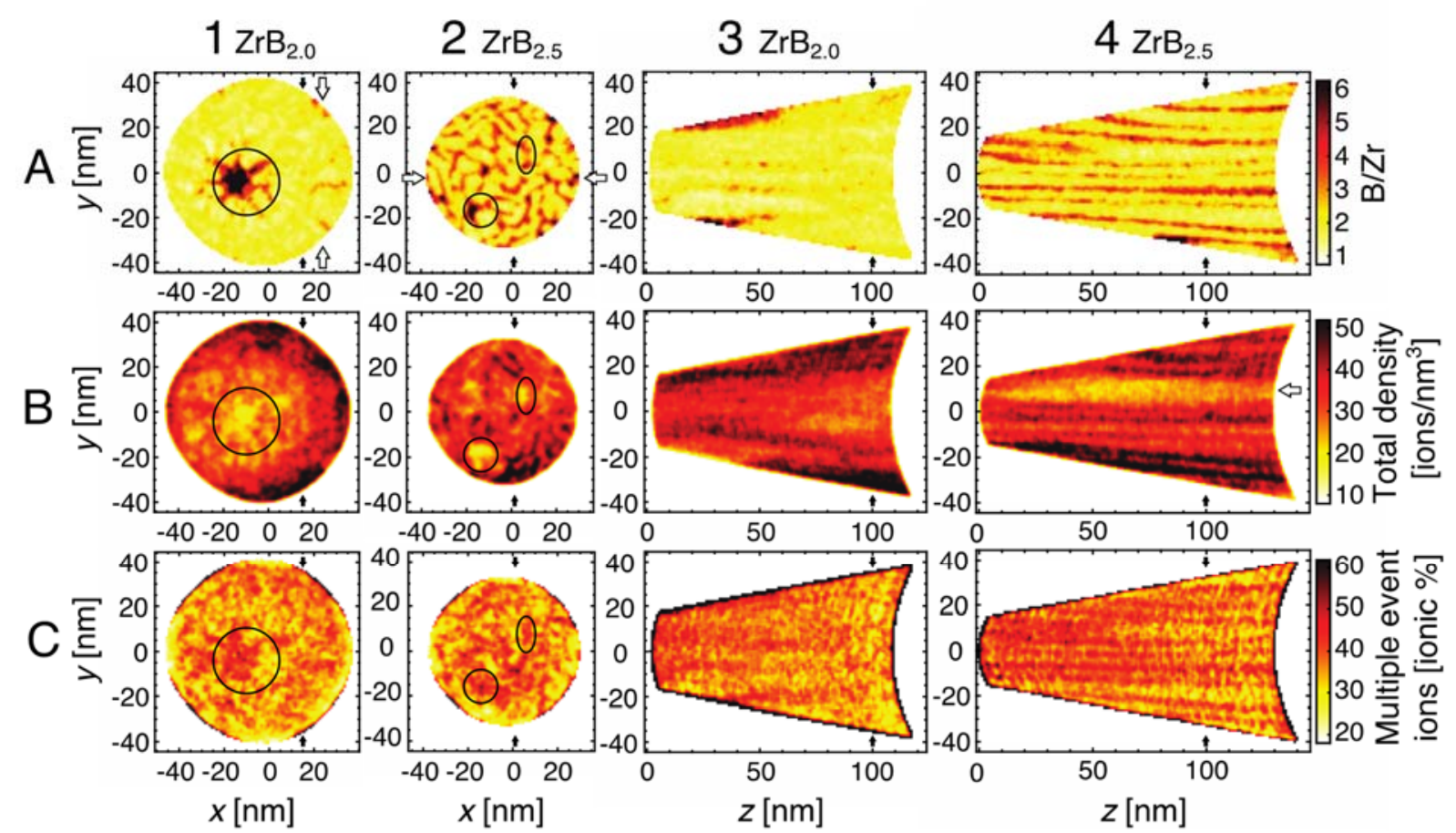

Fig. 7. Uncorrected APT composition maps, total density maps, and multiple event ions maps from $1 \mathrm{~nm}$ thick regions of $\mathrm{ZrB}_{2.0}$ (columns 1 and 3 ) and $\mathrm{ZrB}_{2.5}$ (columns 2 and 4). Regions of interest are marked by circles, ovals and white arrows, while black arrows show the positions of the in-plane maps in the cross-sectional maps and vice versa. The dark color in the outermost regions of the multiple event ions percentage maps is an artefact. The growth direction is opposite to the analysis $(z)$ direction.

The pole region, marked by circles in column 1 of Fig. 7, is the dominating feature in $\mathrm{ZrB}_{2.0}$ and is visible in all three rows. It is the result of inhomogeneous electric field distribution related to the specimen crystallography [74]. Based on the high B/Zr ratio in the pole region in $\mathrm{A} 1$, a locally higher field may have resulted in preferential evaporation of $\mathrm{Zr}$, while the causes of the total density reduction in B1 would also include trajectory aberrations away from the crystallographic pole. The same trend as in the pole region, with high $\mathrm{B} / \mathrm{Zr}$, low density and high multiple event ions percentage is, however, visible for both films. An apparent exception to this trend is the stripe of low density in a region with low $\mathrm{B} / \mathrm{Zr}$ ratio, marked with a white arrow in B4, but this is rather caused by the close proximity of a B rich grain boundary marked by ovals in column 2. Traces of a few B-enriched boundaries in $\mathrm{ZrB}_{2.0}$ can be seen outside the pole region in $\mathrm{A} 1$ and $\mathrm{A} 3$.

In $\mathrm{ZrB}_{2.5}$, there are regions of high $\mathrm{B} / \mathrm{Zr}$ that seem to confine narrow regions with compositions close to stoichiometric $\mathrm{ZrB}_{2}$, like a continuous network around the sides of 
$\mathrm{ZrB}_{2}$ columns. In this structure, a few regions stand out and the most prominent example is marked by circles in column 2 of Fig. 7. This could potentially constitute a pole, but since no repetitive c-planes are found and there is barely any angular mismatch between the grains and the low-density region, in opposite of the case in the $x$-direction of the pole in $\mathrm{ZrB}_{2.0}$ (as can be seen in the supplementary video of $\mathrm{B} / \mathrm{Zr}$ in $\operatorname{ZrB} 2.0$ along $y$ ), this is likely due to an actual change in composition and chemistry at the intersection of several boundaries, rather than field distortion at a low index pole [74].

The multiple event ions maps in row $\mathrm{C}$ are created by dividing the total multiple event ion densities with the total ion densities. Even though the sampling parameters were the same, the sampling itself was not identical for the two densities. In addition, the total ion density is very low at the edges of the analysis. These two facts combined resulted in unreasonably dark edges in the row $\mathrm{C}$.

$\mathrm{B} / \mathrm{Zr}$ profiles from $5 \times 5 \mathrm{~nm}$ cuboids across grains and boundaries in regions shown by white arrows in A1 and A2 of Fig. 7 are presented in Fig. 8. For ZrB2.0, the ratio fluctuates between 1.5 and 2.0, with distinct boundaries between 2.7 and 3.0. In $\mathrm{ZrB}_{2.5}$, the ratio is also 1.5 to 2.0 , narrow boundaries or combinations of grain and boundary have ratios from 2.3 up to 3.0 , while distinct boundaries reach up to 4.5 , excluding the peak at 5.0 found near the reconstruction edge.

a

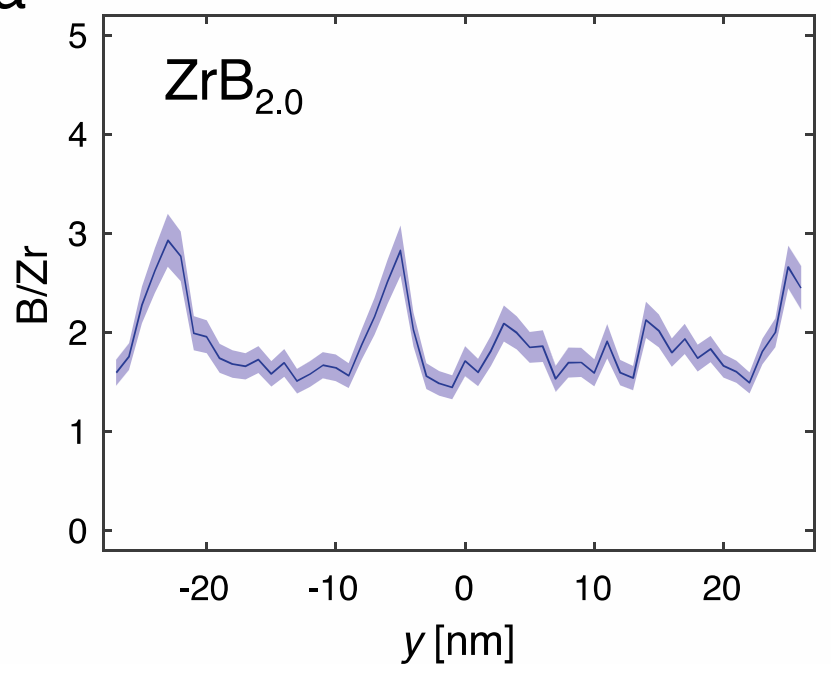

b

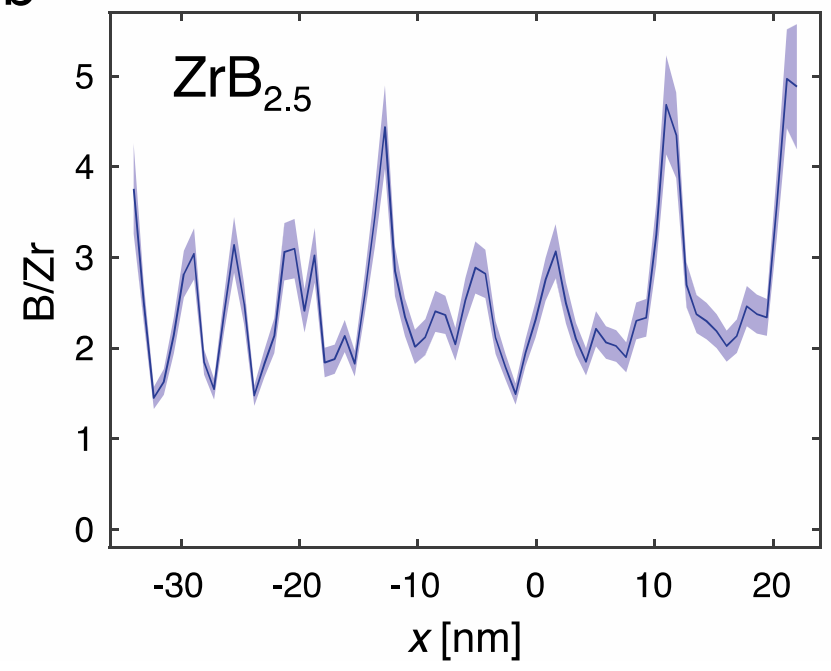

Fig. 8. B/Zr line profiles from $5 \times 5 \mathrm{~nm}$ cuboids in a) $\mathrm{ZrB}_{2.0}$ and b) $\mathrm{ZrB}_{2.5}$ with shaded area representing $1 \sigma$ error bars from counting statistics. The approximate positions of the cuboids are marked by white arrows in a) A1 and b) A2 of Fig 7, centered at the same depth $(z)$ as the maps. 
With the compositional distribution revealed, the three compositional correction procedures in Section 3.5 were applied to the APT data using the isotopic signature from the natural abundance for $\mathrm{Zr}^{3+}$ and five different isotope ratios for $\mathrm{B}$. These were from the natural abundance, the ratios measured by ERDA (Table 1), ratios calculated using Poisson statistics on single-shot APT data, as described by Stephan et al. [75], ratios measured in the co-evaporation correlation scatter plots (of which one is shown in Fig. 6) for $\mathrm{B}$ ion pairs of different charge states (e.g. ${ }^{10} \mathrm{~B}^{+} /{ }^{10} \mathrm{~B}^{2+}$ at $x=5 \mathrm{Da}, y=10 \mathrm{Da}$ ) and lastly ${ }^{10} \mathrm{~B}^{3+} /{ }^{11} \mathrm{~B}^{3+}$ measured in the respective mass spectrum. The first is easily available in literature, but may or may not be related to the actual B isotope ratio in the investigated specimens. The ratio from ERDA is expected to be among the best, as this is measured directly in the investigated films, but it would be convenient if ERDA is not required for future use of the correction procedures. The latter three are measured not only in the investigated film, but in the actual specimen used. Since $\mathrm{B}^{3+}$ ions are rare compared to $\mathrm{B}^{+}$and $\mathrm{B}^{2+}$ in the APT analyzes of this study, the likelihood of generating $\mathrm{B}^{3+}$ pairs should be low and thus less affected by the detector dead-time/space, however, the peaks may not have enough ions to correctly represent the actual isotope ratio, depending on the analysis parameters used and the total number of reconstructed and ranged ions. In the case of multiple event ion pairs from different charge states, it is unknown how well the ratio correlates with the actual B isotope ratio of the specimen.

The results of the compositional corrections are found in Table 2. Based on the accuracy of the ${ }^{10} \mathrm{~B}$ - and combined corrections, measuring the $\mathrm{B}$ isotope ratio using the $\mathrm{B}^{3+}$ mass spectral peaks or ion pairs in the co-evaporation correlation scatter plots where the ions have different charge states cannot be recommended. After excluding these $\mathrm{B}$ isotopes ratios, the maximum $\mathrm{B} / \mathrm{Zr}$ difference is only 0.02 for pile-up pairs

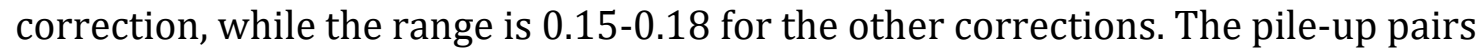
correction must thus be less dependent on knowing the exact isotope abundance, which results in higher precision. Since the pile-up pairs correction using the B isotope ratio estimated by Poisson statistics on single-shot APT data seems to have good precision and accuracy, it will be considered further below. The corrected B/Zr ratios of the APT specimens are thus 2.0 for $\mathrm{ZrB}_{2.0}$ and $\sim 2.7$ for $\mathrm{ZrB}_{2.5}$. 


\begin{tabular}{|c|c|c|c|}
\hline & $\begin{array}{c}\text { B isotope ratio } \\
\text { source }\end{array}$ & $\begin{array}{c}\mathrm{ZrB}_{2.0} \\
\text { (Pole removed) }\end{array}$ & $\mathrm{ZrB}_{2.5}$ \\
\hline ERDA reference & - & 1.99 & 2.48 \\
\hline APT Uncorrected & - & 1.83 & 2.40 \\
\hline \multirow{5}{*}{$\begin{array}{l}\text { APT } \\
\text { Pile-up pairs } \\
\text { Correction }\end{array}$} & ${ }^{10} \mathrm{~B}^{3+} /{ }^{11} \mathrm{~B}^{3+}$ & 2.06 & 2.74 \\
\hline & Natural & 2.04 & 2.69 \\
\hline & ERDA & 2.03 & 2.68 \\
\hline & Single-shot APT & 2.02 & 2.67 \\
\hline & 2 charge states & 2.00 & 2.62 \\
\hline \multirow{5}{*}{$\begin{array}{l}\text { APT } \\
{ }^{10} \text { B-method }\end{array}$} & ${ }^{10} \mathrm{~B}^{3+} /{ }^{11} \mathrm{~B}^{3+}$ & 2.31 & 3.24 \\
\hline & Natural & 2.11 & 2.82 \\
\hline & ERDA & 2.04 & 2.74 \\
\hline & Single-shot APT & 1.95 & 2.65 \\
\hline & 2 charge states & 1.73 & 2.25 \\
\hline \multirow{5}{*}{$\begin{array}{l}\text { APT } \\
\text { Iterative }{ }^{10} \mathrm{~B}- \\
\text { method }\end{array}$} & ${ }^{10} \mathrm{~B}^{3+} / 11 \mathrm{~B}^{3+}$ & 2.38 & 3.35 \\
\hline & Natural & 2.15 & 2.88 \\
\hline & ERDA & 2.07 & 2.78 \\
\hline & Single-shot APT & 1.97 & 2.68 \\
\hline & 2 charge states & 1.72 & 2.23 \\
\hline APT & ${ }^{10} \mathrm{~B}^{3+} /{ }^{11} \mathrm{~B}^{3+}$ & 2.36 & 3.30 \\
\hline \multirow[t]{4}{*}{ Combined } & Natural & 2.15 & 2.88 \\
\hline & ERDA & 2.09 & 2.80 \\
\hline & Single-shot APT & 2.00 & 2.71 \\
\hline & 2 charge states & 1.78 & 2.31 \\
\hline
\end{tabular}

Table 2. Uncorrected and corrected average B/Zr of $\mathrm{ZrB}_{2.0}$ and $\mathrm{ZrB}_{2.5}$ from APT reconstructions using several correction procedures and B isotope ratios, compared to the ERDA reference values from Table 1.

\subsection{Trace elements}

Atom probe tomography is perfect for measuring and mapping trace elements in small volumes due to its low detection limit and high resolution in 3-D. The mass spectra in Fig. 3 reveal that there are trace amounts of many metals, as well as a few metalloids and non-metals. The impurities (excluding $\mathrm{H})$ are $\mathrm{C}(<0.5$ at. \%), $\mathrm{O}$ and $\mathrm{Hf}(<0.4$ at. \%), $\mathrm{Al}$, and $\mathrm{Ar}(<0.2$ at. \%), as well as $\mathrm{N}, \mathrm{Fe}, \mathrm{Ti}, \mathrm{As}, \mathrm{Cr}$, and $\mathrm{Mn}(<0.1$ at. \%). Hf is an expected impurity due to the difficulties of separating Hf from Zr during metallurgical fabrication 
[76]. The other metals and metalloids are likely also from the target, since all but Cr are reported as common impurities by the supplier, while the non-metals may have different origins. The presence of Ar is explained by the use of Ar gas in the plasma during film growth, while it is likely that $\mathrm{C}, \mathrm{N}$, and $\mathrm{O}$ at least partly come from the compound cathode, as was discussed for $\mathrm{C}$ and $\mathrm{O}$ in [45]. The probable source of $\mathrm{H}$ is the residual gas in the APT analysis chamber, since $\mathrm{H}$ and $\mathrm{H}_{2}$ were homogeneously distributed in APT, and ERDA showed that there are insignificant amounts of $\mathrm{H}$ within the films.

The distributions of $\mathrm{Hf}, \mathrm{Al}$, and Fe are clearly correlated with regions of higher than average $\mathrm{Zr}$ in $\mathrm{ZrB} 2.5$, while this tendency could not be confirmed for the remaining metals and metalloids. The presence of $\mathrm{Hf}$ and Al suggests substitutional alloying [77] in the metal sub-lattice, as both form $\mathrm{AlB}_{2}$-type crystal structure. Ascertaining the correlation of the non-metals individually is difficult, however, once combined it is evident that they are more likely found in regions of higher than average B. Regions of either high metal and metalloid trace elements or high non-metal trace elements also exist in $\mathrm{ZrB}_{2.0}$.

The ERDA and APT analyses coincide well on the Ar content, while $\mathrm{N}$ and $\mathrm{O}$ are slightly lower in APT, possibly due to differences in probed volume. The $\mathrm{C}$ content was not quantified by ERDA, but is fairly low in APT, even though it is the most common trace element. Compared to surface sensitive XPS of an epitaxial stoichiometric $\mathrm{ZrB}_{2.0}$ film, the $\mathrm{O}$ contamination measured by APT and ERDA, as well as the $\mathrm{C}$ content measured by APT alone, are significantly lower (about 4 at. \% and 1.5 at. \% lower, respectively) even though sputter-cleaning was conducted before the XPS analysis [41]. This is consistent with previously reported discrepancies between ERDA and XPS analyses of $\mathrm{ZrB}_{2}$ on $4 \mathrm{H}-\mathrm{SiC}(0001)[45,78]$, where the high oxygen content in XPS is attributed to difficulties with sputter-cleaning of surface oxides due to high reactivity of 0 toward Zr.

\subsection{Grain stoichiometry and boundaries}

The exact stoichiometry of the grains is of interest, but this is difficult to determine with APT due to the detector dead-time/space. The correction procedures in Section 3.5 provide factors for correcting the composition of the entire specimen, but it is unclear whether this will allow determining the stoichiometry of, e.g., a single grain or column. 
Because of this, the average stoichiometry of 15-18 columnar regions for each of the two specimens shown in Fig. 7 are investigated instead. The regions were enclosed by cylindrical volumes with lengths at small angles to the direction of the analysis $(z)$. As metallic and metalloid trace elements tend to be found in Zr-rich regions, while nonmetal trace elements are most abundant in B-rich regions, trace element iso-surfaces combined with $\mathrm{Zr}$ ratio iso-surfaces were used to identify regions of interest. Since the size of the columns differed, the diameters, $d$, of the elliptical cross sections were set manually for each column. The diameters of all elliptical cylinders were then adjusted in steps of $\pm 2 \mathrm{~nm}$, although the minimum resulting diameter $d+\Delta d$ was set to $1 \mathrm{~nm}$, which affects a few of the smallest cylinders in the transition from $\Delta d=-2$ to $\Delta d=-4 \mathrm{~nm}$ only, i.e., no cylinders are removed completely even if a manually assigned diameter $d$ is $4 \mathrm{~nm}$ or less. By investigating the ratio of B and $\mathrm{Zr}$ atoms in each cylinder after subtracting the atoms in the slightly smaller cylinder, the cylindrical radial distribution functions in Fig. 9 were generated. The standard deviation for B/Zr from counting statistics derived using the formula by Danoix et al. [79] for local concentrations was always $<0.025$, which corresponds to the marker size.
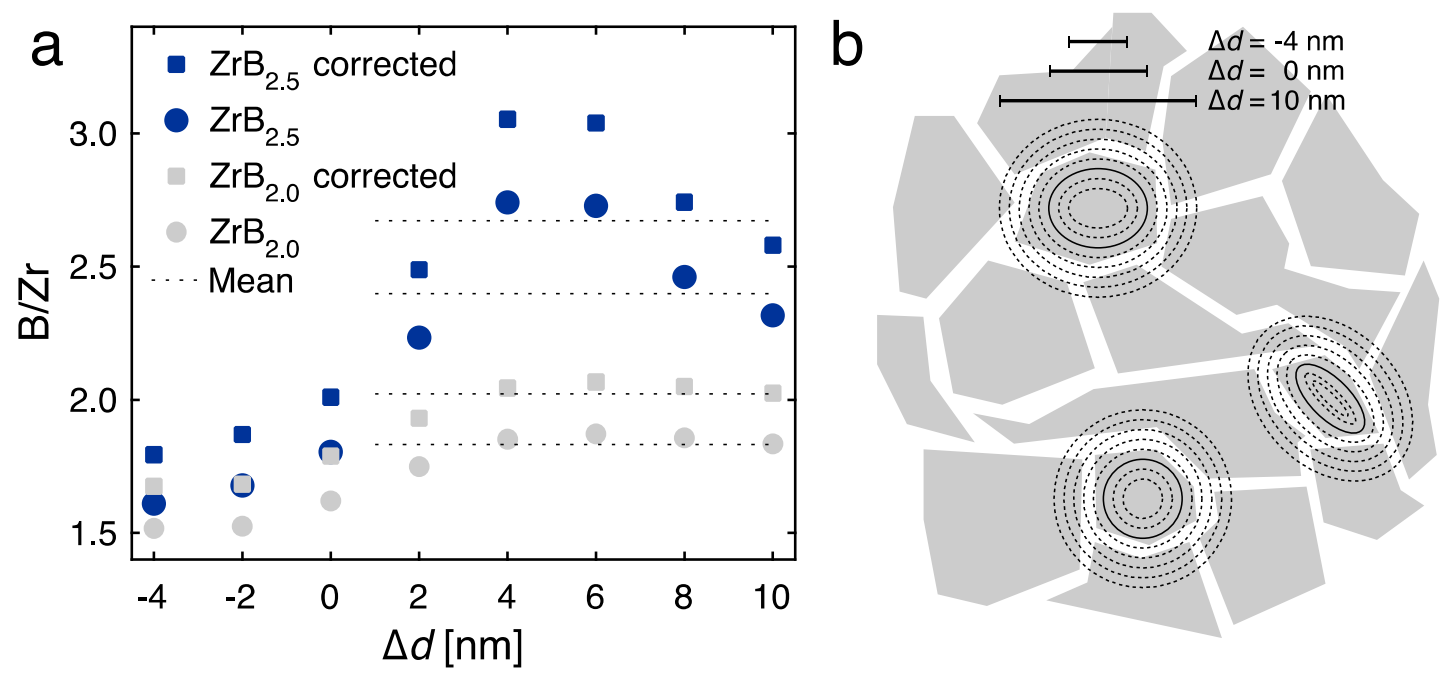

Fig. 9. Cylindrical radial distribution showing a) the B/Zr ratio in cylinders (at $\Delta d=-4$ $\mathrm{nm}$ ) and cylindrical shells (at $\Delta d \neq-4 \mathrm{~nm}$ ) of different sizes aligned along Zr-rich columns at small angles to the growth direction and b) a schematic representation of columns (grey), boundaries (white) and three out of the 15-18 analyzed regions (ellipses). Manually assigned elliptical cylinders, $\Delta d=0 \mathrm{~nm}$, are drawn with solid lines. 
The uncorrected values are multiplied by a correction factor determined by pile-up pairs correction using the B isotope ratio estimated by Poisson statistics on single-shot APT data. In $\mathrm{ZrB}_{2.0}$, the pole region was removed before the factor was calculated and no cylinders were positioned there.

The B/Zr-ratio in the smallest cylinders of Fig. 9 was lower than the value for stoichiometric $\mathrm{ZrB} 2$ and fairly similar for both compositions. As the cylinders become cylindrical shells and grow larger, the B/Zr reaches a maximum when the shell outer diameter $d$ is $\sim 5 \mathrm{~nm}$ larger than that of the manually assigned cylinders, after which the ratios approach the specimen average compositions as measured by APT and, in the case of $\mathrm{ZrB}_{2.5}$, even decrease below it.

\subsection{Electrical resistivity}

The in-plane electrical resistivity of the two films was measured using a four-point probe, in order to study the influence of the microstructure. The resistivity of $\mathrm{ZrB}_{2}$ thin films has previously been studied as a function of substrate bias voltage [78], annealing time [80], argon pressure [81], deposition temperature [45], sputtering power [45,81], and pulse repetition frequency in high-power impulse magnetron sputtering (HiPIMS) film growth [82]. The resistivity of the films in this study was $160 \mu \Omega \mathrm{cm}$ for $\mathrm{ZrB} 2.0$ and $390 \mu \Omega \mathrm{cm}$ for $\mathrm{ZrB}_{2.5}$. This is an increase by a factor of 2.4 in $\mathrm{ZrB}_{2.5}$ compared to $\mathrm{ZrB}_{2.0}$ and both values are within the range of previous measurements of as-deposited magnetron sputtered $\mathrm{ZrB}_{2}$ films from compound targets.

Although the electrical resistivity of $\mathrm{ZrB}_{2}$ can depend on many parameters, such as crystal quality, presence of additional grain orientations, and 0 content $[45,83]$, one likely source of the increase in electrical resistivity in $\mathrm{ZrB}_{2.5}$ compared to $\mathrm{ZrB}_{2.0}$ is the continuous and wide B-rich column boundaries in $\mathrm{ZrB}_{2.5}$. While the graphene-like B layers perpendicular to the 0001 direction are responsible for the low in-plane resistivity of the iso-structural $\mathrm{MgB}_{2}$ [84], the $\mathrm{Zr}$ layers are the source of the relatively low in-plane resistivity of $\mathrm{ZrB}_{2}$ [85]. Since the B-rich boundaries break the lateral extent of the $\mathrm{Zr}$ layers, and most likely the B layers as well, the in-plane resistivity is increased. If the B-rich boundaries are the main reason for the increased resistivity, films close to stoichiometric $\mathrm{ZrB}_{2}$ would be better suited for wear resistant electrical contacts, but a study of the resistivity in several films with boundaries of different widths is required to confirm this. 


\section{Discussion}

\subsection{Field evaporation behavior}

Field evaporation of ceramics is seldom ideal enough to produce the hemispherical equilibrium surface assumed by most APT reconstruction algorithms. Correlative evaporation behavior [53] is common, which results in comparatively high multiple events percentages even at low evaporation rates, although, as shown in Fig. 4, $\mathrm{ZrB}_{2}$ has a relatively low multiple events percentage and thus seems to be better in that regard compared to many other hard ceramic films. A possible reason for this could be the $\mathrm{AlB}_{2}$ type crystal structure of the concerned films. The unit cell of $\mathrm{ZrB}_{2}$ is shown in Fig. $10 \mathrm{a}$ ) and $b$ ). This structure grown in the orientation of the present films results in alternating layers of honeycomb, graphene-like B layers and hexagonally close packed Zr layers at small angles to the growth and analysis direction ( $z$ ), as visualized in Fig. $10 \mathrm{c}$ ). B-B bonds are covalent and strong, while the metallic $\mathrm{Zr}-\mathrm{Zr}$ bonds and combined covalent and ionic Zr-B bonds are significantly weaker $[42,85]$. Since field evaporation of atoms on top of a layer generally requires less energy than breaking B-B and Zr-Zr bonds within a complete layer, a subsequent layer-by-layer field evaporation may be promoted by the crystal structure and orientation of the grains in this study, although the polycrystalline nature of the film will likely prevent the formation of continuous $B$ and $\mathrm{Zr}$ layers throughout the entire width of the specimen. Stacking faults at semi-coherent boundaries have been reported for $\mathrm{ZrB}_{2.0}$ [44], and the wider boundaries of $\mathrm{ZrB}_{2.5}$ likely separate such layers even more.

The distribution of false single ions and multiples with artificially low multiplicity (and thereby also the distribution of the ions lost due to detector dead-time/space) is impossible to know, and will thus limit the local accuracy of the correction procedures. The detected multiple events percentage might provide clues as to where most ions are lost. While it could be argued that the lower percentage of detected multiple event ions is the result of ion loss and that most lost ions were field evaporated from such regions, the estimated number of lost ions constitute $<9 \%$ of all multiple event ions, and can thus not account for the strong local difference in multiple event ions percentage clearly seen in row $\mathrm{C}$ of Fig. 7, even though the total ion density is lower in these regions. It is more likely that regions with high multiple events percentage also have higher ion losses, but that the losses have marginal effect on the difference in the local distribution 
of multiple events. As seen by comparing row A and C of Fig. 7, the percentage of multiple event ions was higher in regions of high B/Zr and low density.

a

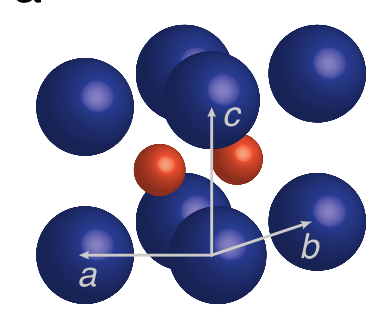

b

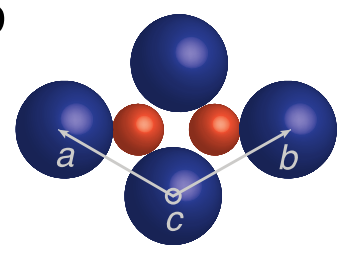

C

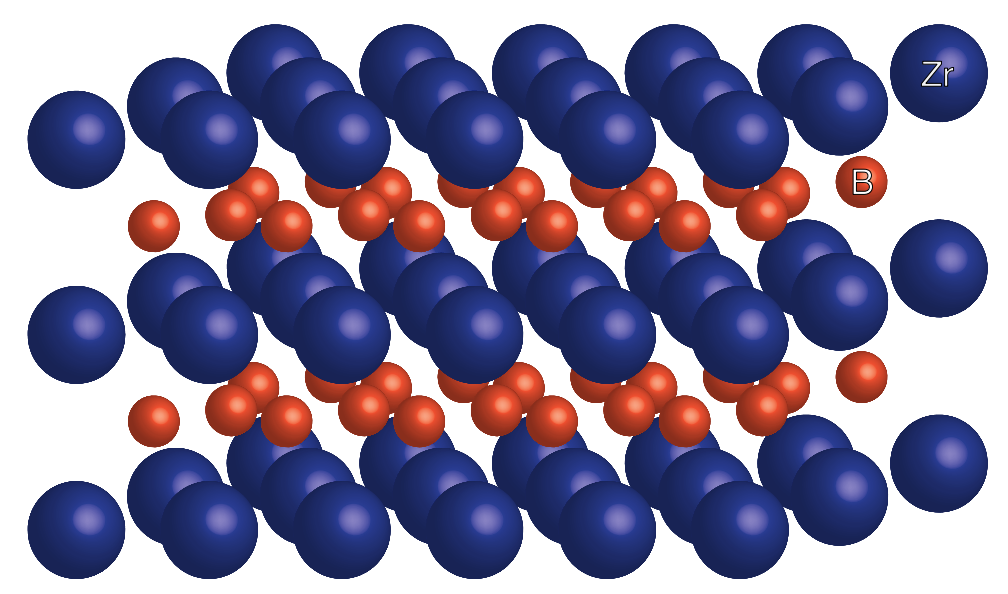

Fig. 10. The unit cell of the $\mathrm{AlB}_{2}$-type crystal structure formed by $\mathrm{ZrB}_{2}$ in a) side view and

b) along to the surface normal of the c) graphite-like B layers and hexagonally close packed layers of $\mathrm{Zr}$. The distance in the $c$-direction (0001) has been increased in c) to reveal the alternating $\mathrm{B}$ and $\mathrm{Zr}$ layers. The unit cell contains one $\mathrm{Zr}$ atom and two B atoms.

Signs of preferential B retention in $\mathrm{ZrB}_{2}$ were found in the very start of the analyzes, as shown for $\mathrm{ZrB}_{2.5}$ in Fig. 5. The curve starts at B/Zr of 0.5 and with a sharp peak at $z=4$ $\mathrm{nm}$. The initial low value suggests that $\mathrm{Zr}$ is field evaporated first while B is preferentially retained. Shortly after this, a B/Zr maximum is reached as B is overrepresented on the specimen surface when most available $\mathrm{Zr}$ has been field evaporated. Eventually, the shape of the specimen apex has been transformed into an equilibrium surface, where the probability of evaporation from any part of the specimen apex is the same (on a scale larger than the atomic scale), and a B/Zr steady state is reached. The equilibrium surface is, however, not hemispherical, as the reconstruction algorithm assumes. Preferentially retained B at the boundaries results in B-enriched protrusions from where (correlated) field evaporation eventually occurs. Field evaporated atoms from protrusions will suffer from trajectory aberrations that locally magnifies these regions, leading to ion spreading and a reduction in measured local density [86-88] that can clearly be seen in row B of Fig. 7. If correlated field evaporation occurs, this results in more multiple detection events and ion losses due to the detector dead-time/space. All this suggests that relatively more B is lost in regions of high $\mathrm{B} / \mathrm{Zr}$ 
with locally higher multiple events percentage. However, correlated evaporation will still occur from grains, probably to a greater extent when the center of a new layer is penetrated [53] since this changes the bonding of atoms next to the newly formed surface vacancy on the specimen apex. Both correlated evaporation and preferential retention change the order in which the ions are field-evaporated, thereby degrading the depth $(z)$ resolution [89]. In addition, it is more likely that preferentially retained atoms migrate on the specimen apex prior to being field-evaporated, thus also degrading the lateral $(x, y)$ resolution of retained species [90].

This study has clearly shown that the field evaporation behavior of $\mathrm{ZrB}_{2}$ is far from ideal and suffers from several aberrations. While aberrations in general are undesired, some may also be useful, such as the pole artefact for estimating reconstruction parameters from the lattice spacing along 0001 or local magnification for identifying phases with different evaporation field in the total ion density distribution.

\subsection{Compositional correction of APT reconstructions}

An Imago LEAP 3000X HR atom probe was used in this study. Even though the multi-hit detection algorithm has been improved in newer models of the LEAP [91], the actual improvement is more or less negated by the increase in detection efficiency [68,92], since this increases the likelihood of multiple detector events [93]. Using the current detector technology with materials systems that exhibit correlative field evaporation behavior will always lead to false single ions and multiple event ions with artificially low multiplicity, i.e., single or multiple ion detections of fewer ions than what hit the detector. The ion loss must be corrected in order to avoid compositional shifts and thereby increase the accuracy of the average (and preferably also local) composition measured by APT.

In Table 2, several correction methods and ${ }^{10} \mathrm{~B} /{ }^{11} \mathrm{~B}$ ratios are compared with each other and with the average compositions from ERDA. First, the different ratios will be considered. Two ratios yield greatly inaccurate values of $\mathrm{B} / \mathrm{Zr}$ with the ${ }^{10} \mathrm{~B}$ - and combined methods. In the co-evaporation correlation data, ${ }^{10} \mathrm{~B} /{ }^{11} \mathrm{~B}>0.3$ is found when using B-pairs with charge states one and two. With a ${ }^{10} \mathrm{~B} /{ }^{11} \mathrm{~B}$ ratio higher than the reconstruction average, the adjustment of the number of ${ }^{11} \mathrm{~B}$ ions to reflect the number of ${ }^{10} \mathrm{~B}$ ions, as is done in the ${ }^{10} \mathrm{~B}$ - and combined methods, will result in a reduction of the number of ${ }^{11} \mathrm{~B}$ ions, which contradicts the higher loss of ${ }^{11} \mathrm{~B}$ in Fig. 3 and Fig. 6. Since 
correction using this abundance also gives $\mathrm{B} / \mathrm{Zr}$ ratios below the uncorrected APT and ERDA results, it must be excluded. Measuring ${ }^{10} \mathrm{~B} /{ }^{11} \mathrm{~B}$ from $\mathrm{B}^{3+}$ in the mass spectra of Fig. 3 gives ${ }^{10} \mathrm{~B} /{ }^{11} \mathrm{~B} \sim 0.2$. As expected, this charge state is not as heavily affected by the detector dead-time/space due to its scarcity in this study. The low ratio found lead to excessive enumeration of $\mathrm{B}$. The likely cause of the low ratio is counting statistics uncertainty, with ${ }^{10} \mathrm{~B}^{3+}$ counts as low as 2245 , and possibly by a difference in the isotopic signature of $\mathrm{B}^{3+}$, as was reported for $\mathrm{B}^{+}$and $\mathrm{B}^{2+}$ in $\mathrm{CoB}$ and $\mathrm{FeB}$ [94]. Given the great difference in B/Zr from both the ERDA reference and the APT values corrected with the ${ }^{10} \mathrm{~B}$ - and combined methods using other abundances, the $\mathrm{B}$ isotope ratio from $\mathrm{B}^{3+}$ should be disregarded. The $\mathrm{CoB}$ and FeB abundances from [94] were also used with the correction procedures, but produced insignificant differences from the natural abundance and are thus not presented in Table 2. The remaining three (natural, ERDA, and single-shot APT) B isotope ratios seem to yield reasonably small B/Zr differences, with the ratio from Poisson statistics of single-shot APT data having the highest accuracy with regards to the ERDA references.

The correction procedures will now be considered. As expected, the combined method yielded $\mathrm{B} / \mathrm{Zr}$ ratios slightly higher than the ${ }^{10} \mathrm{~B}$-method, but comparable to those of the iterative ${ }^{10} \mathrm{~B}-$ method. The difference between the ${ }^{10} \mathrm{~B}$-method and the iterative ${ }^{10} \mathrm{~B}$ - or combined method should be greater when the multiple events percentage is higher and more ${ }^{10} \mathrm{~B}$ ions are lost. The $\mathrm{B} / \mathrm{Zr}$ value corrected with the pile-up pairs method was generally lower than B/Zr corrected by other methods. One important exception is when the B isotope ratio from single-shot APT data is applied to $\mathrm{ZrB}_{2.0}$, where the B/Zr value corrected with the conservative pile-up pairs correction was higher than the combined correction. This suggests that the estimated value of ${ }^{10} \mathrm{~B} /{ }^{11} \mathrm{~B}$ might be somewhat too high, resulting in lower $\mathrm{B} / \mathrm{Zr}$ for the ${ }^{10} \mathrm{~B}$ and combined corrections. Compared to other sources of errors, the B/Zr difference of 0.02 between the combined and pile-up pairs corrections is insignificant, but enough to warrant using the pile-up pairs corrected value instead, even though the value from the combined correction is the closest to the ERDA reference.

From the small B/Zr difference in Table 2 when using the pile-up pairs correction (even with the discarded isotope ratios), it is evident that this correction has the highest precision and is not as dependent on knowing the exact abundance as the ${ }^{10} \mathrm{~B}$ - and combined methods, since the correction is applied to fewer ions in total. It is thus the 
best choice when there are uncertainties regarding the isotope ratio, while keeping in mind that the correction is generally conservative.

In the case of $\mathrm{ZrB}_{2.0}$, the low-density (pole) region was excluded before compositional correction, since Zr may have been lost due to preferential evaporation. This resulted in a reduction of $\mathrm{B} / \mathrm{Zr}$ for all values in the $\mathrm{ZrB}_{2.0}$ column of Table 2, except the ERDA reference. Similar exclusion of low-density regions in $\mathrm{ZrB} 2.5$ had marginal effect on $\mathrm{B} / \mathrm{Zr}$. This suggests that the low-density regions in $\mathrm{ZrB}_{2.5}$ are not pole regions, possibly not even crystalline, which is confirmed by the lack of detectable lattice distances in these regions. In $\mathrm{ZrB}_{2.0}$ with the pole removed, $\mathrm{B} / \mathrm{Zr}$ values from all correction methods are found within \pm 0.1 from the ERDA reference when using $B$ isotope ratio from ERDA and within \pm 0.04 when using the ratio from Poisson statistics on single-shot APT data. As this specimen is relatively homogenous, it is the best measure of accuracy in this study. The difference in B/Zr between the ERDA reference and corrected APT was greater in $\mathrm{ZrB}$ 2.5. Given the excellent result in $\mathrm{ZrB} 2.0$, this discrepancy is likely due to differences in the probed volume between ERDA and APT, and not a flaw of the correction procedures. To better determine the accuracy of the correction methods, they should be tested further on several homogeneous reference samples with a large span of well-determined compositions, preferably either single crystal or very small-grained, so as to remove or average out the influence of grain boundaries, although single crystals will likely produce pole artefacts.

Based on ERDA and the corrected APT results, the pile-up pairs correction is the best choice for compositional correction of $\mathrm{ZrB}_{2}$, as it is less dependent on background correction, knowing the exact isotopic abundances, and not prone to over-estimate the corrected elements. With high precision of $\mathrm{B} / \mathrm{Zr}$ using different correction methods and the best accuracy compared to the ERDA reference, the method by Stephan et al. [75] to estimate the B isotope abundance using Poisson statistics on single-shot APT data from each reconstruction seems to be the best choice for acquiring the necessary isotope ratios, especially when the average ratio has not been determined by other techniques, such as ERDA.

To sum up, when applying the correction methods discussed here, the specimens should have a known, or close to natural, isotope abundance, even though the pile-up pairs correction was significantly less sensitive to such errors. The ${ }^{10} \mathrm{~B}$ - and combined methods also require careful background correction. At least two stable isotopes of the 
elements to be corrected is required for finding reference pairs or peaks in all methods discussed here.

\subsection{Film composition}

The compositions measured by ERDA and APT were quite close even without correction ( $<2$ at. \% each of $\mathrm{B}$ and $\mathrm{Zr}$ ), although in the case of the heterogeneous $\mathrm{ZrB} 2.5$, this might be due to differences in probed volume. Even the conservative pile-up pairs correction yielded APT B/Zr ratios higher than the ERDA reference for $\mathrm{ZrB}_{2.5}$. If the sub-volume investigated by APT is not representative for the entire sample, but rather contain a larger fraction of wide, disordered, B-rich grain boundaries and junctions than the much bigger probed volume of the ERDA analysis, the correction procedure could be accurate also for this specimen. For comparison, the B/Zr ratio in a different $\mathrm{ZrB}_{2.5}$ specimen (not shown) was $\sim 2.3$ uncorrected and $\sim 2.6$ corrected (in contrast to 2.4 and $\sim 2.7$, respectively). Regardless of the source of the discrepancy for $\mathrm{ZrB} 2.5$, the correction procedures applied to the $\mathrm{ZrB}_{2.0}$ specimen changes the interpretation of the APT measurement from under-stoichiometric with regards to B to stoichiometric $\mathrm{ZrB}_{2}$ and point toward both good precision and accuracy, well within the $5 \%$ (relative) error range of the ERDA atomic compositions.

\subsection{Grain stoichiometry}

At first glance on row A of Fig. 7, it looks like $\mathrm{ZrB}_{2}$ is a line compound. However, the lower limit of $\mathrm{B} / \mathrm{Zr}$ in Fig. 8 reveals that there are regions of $\mathrm{B} / \mathrm{Zr}<2$ in the very center of some columns even in the B over-stoichiometric specimen. This is confirmed by Fig. 9, which shows that $\mathrm{B} / \mathrm{Zr}$ in cylinders aligned with such columns may be as low as 1.7 (corresponding to $\mathrm{Zr}_{0.37} \mathrm{~B}_{0.63}$ ) even after correction. It must be considered that this regards the smallest cylinders with the least number of ions in regions found through iso-concentration surfaces that are very sensitive and easily find minute fluctuations in composition. Although the counting statistics error is decreased by the use of many cylinders or cylindrical shells, the very low values of B/Zr may still be an artefact, where counting statistics is one part and insufficient B correction could be another. In the case of $\mathrm{ZrB}_{2.0}$, it is possible that there is a slight influence of the pole even outside the excluded region, as the region with seemingly affected density in B1 of Fig. 7 is larger than the region with clearly affected B/Zr in A1 of Fig 7. With increasing relative 
cylinder diameters, the number of ions included increases, and at some point, the cylindrical shells reach out into the grain boundaries, where the B/Zr increases to $\sim 2$ in $\mathrm{ZrB}_{2.0}$ and $\sim 3$ in $\mathrm{ZrB} 2.5$. Eventually, the cylindrical shells become so large that they are found outside the nearest boundaries, which results in $\mathrm{B} / \mathrm{Zr}$ closer to the average value measured by APT.

Neither of the correction procedures applied in this study can reveal the 3-D positions of the lost ions, but as discussed in Section 4.1, the distribution of multiple events may provide a hint to where the ions are lost. Since it is likely that a majority of the lost B ions originated from the B-rich boundaries, where the percentage of ions from multiple events is high, the homogeneously applied correction factor based on the average composition before and after corrections, as in this paper, would on one hand increase the B content of the columns with more than what was lost, and the boundaries with less than was lost. On the other hand, the risk of overestimating the B content of the grains is reduced by using a conservative correction method, such as the pile-up pairs correction used in Fig. 9. The density maps in row B of Fig. 7 reveal that high B/Zr results in low density, because of trajectory aberrations from B-rich protrusion. At the same time, the columns are focused, resulting in higher density. It is thus unlikely that the $\mathrm{B} / \mathrm{Zr}<2$ in the columns is the result of trajectory aberrations, as $\mathrm{B}$ from the boundaries are spread into the focused columns, while the width of the B-rich boundaries is likely overestimated [95].

Even line compounds in phase diagrams have some compositional spread. The phase boundaries are often determined using X-ray analysis with compositional uncertainty of $\sim 1$ at. $\%$. Compounds with smaller compositional spread than 1 at. \% can thus be thought of as line compounds [20]. Even though the APT may not have measured the exact stoichiometry of the grains, the radial compositional gradient within the columnar regions investigated in Fig. 9, with at most 4 at. \% deviation from stoichiometry within the columns toward B sub-stoichiometry, suggests that $\mathrm{ZrB}_{2}$ is a phase with a limited compositional range of at least a few at. \% under non-equilibrium conditions, and thus wider than what previously was reported for equilibrium condition [19]. Since the distribution of multiple events points toward grains being corrected with slightly to much B, insufficient correction is unlikely the cause, as the average corrected APT composition is higher than the ERDA reference. 


\subsection{Grain boundaries}

The Z-contrast STEM images in Fig. 2 b) show that at least two different types of boundaries are present in $\mathrm{ZrB}_{2}$. One is semi-coherent and stich-like, while the other is much wider and likely disordered. APT reveals in Fig. 9, as well as in A2 and A4 of Fig. 7, that even with B over-stoichiometric films, the composition of the grains is fairly close to stoichiometric $\mathrm{ZrB}_{2}$ and that excess $\mathrm{B}$ is present at the grain boundaries. When $\mathrm{B}$ is plentiful, e.g., in $\mathrm{ZrB}_{2.5}$, the boundaries form a B-rich continuous network around columns consisting of one or a few grains separated by semi-coherent boundaries. The B/Zr maximum at $\sim 5 \mathrm{~nm}$ relative cylinder diameter in Fig. 9 is only significant in the $\mathrm{ZrB}_{2.5}$ specimen, but indicate that the investigated grains in $\mathrm{ZrB}_{2.0}$ may be adjacent to a small number of wide, disordered grain boundaries, or be covered by a thin (1-2 monolayers) or incomplete ( $<1$ monolayer) B tissue phase in the stich-like semicoherent grain boundaries, or both. Such a tissue phase was found in $\mathrm{TiB}_{2}$ [16], but has not been confirmed in $\mathrm{ZrB}_{2}$. Considering the composition maps in $\mathrm{A} 1$ and $\mathrm{A} 3$ of Fig. 7 and the related B/Zr profile in Fig 8. a), the maximum is most likely caused by a few Brich boundaries close to the manually assigned cylinders, even though the presence of a thin or incomplete tissue phase cannot be excluded with certainty.

\subsection{Prospects for APT of other diborides}

This study provides input for the general applicability of APT for the wider range of transition metal diborides. The preferential loss of B in multiple events is an expected difficulty, which is why significant efforts need to be made to mitigate the effects on the composition measured by APT, like the models proposed here for compositional correction. Even though the compositional difference was not severe in this study, due to lower multiple events percentage than expected, the correction was still necessary to change the interpretation of the APT composition in $\mathrm{ZrB}_{2.0}$ from clearly understoichiometric to practically stoichiometric.

The experiences from this study are also presently applied to APT of the more complex $\mathrm{ZrTaB}_{2}$ materials system [12], where the primary concern is $\mathrm{Ta} /(\mathrm{Zr}+\mathrm{Ta})$, which should not be as heavily affected by losses due to detector dead-time/space. These samples also consists of columnar 0001-oriented grains with $\mathrm{AlB}_{2}$ crystal structure and the field evaporation produces low-density pole regions with detectable interplanar spacing in the 0001 direction, although with less clarity than in pure $\mathrm{ZrB}_{2}$. 
Further studies of $\mathrm{AlB}_{2}$-structured films with larger angles between the 0001 and the analysis direction might generate more information regarding the evaporation behavior with respect to the alternating metal and B layers with surface normals along the 0001 direction in the $\mathrm{AlB}_{2}$-type crystal structure.

\section{Conclusions}

Atom probe tomography has been applied to two $\mathrm{ZrB}_{2}$ thin films of different $\mathrm{B} / \mathrm{Zr}$ ratios. The field evaporation behavior, as well as its effect on the measured composition, has been investigated and several compositional corrections procedures have been compared with the average composition from ERDA. Stoichiometric $\mathrm{ZrB}_{2.0}$ was found to exhibit a pole artefact with detectable lattice planes perpendicular to the 0001 direction. The lattice distance was used in an iterative reconstruction procedure, which was refined so as to avoid errors from interference in spatial distribution maps between the lattice planes and alternating sparse and dense hemispherical shells from correlated evaporation.

The APT analyses exhibit signs of preferential retention, correlated evaporation, and compositional shift due to the detector dead-time/space. A new pile-up pairs compositional correction procedure for elements with at least two isotopes, based on co-evaporation correlation data, was tested against two versions of the B equivalency of the ${ }^{13} \mathrm{C}$-method and a combination of both methods, in order to improve measurements of the B/Zr ratio. Although the pile-up pairs method (and thus also the combined method) is less trivial to apply than the ${ }^{10} \mathrm{~B}$-methods, and has not yet been applied to coevaporation correlation data with long traces from mutual evaporation delays, a significant increase in precision was achieved, regardless of the accuracy of the isotope abundances used. In addition, the procedure is not influenced by the background correction (or lack thereof), as is the case of the ${ }^{10} \mathrm{~B}$ - and combined methods. The highest accuracy of the average $\mathrm{B} / \mathrm{Zr}$ ratio with respect to the ERDA reference was achieved using the B isotope ratio estimated by Poisson statistics from single-shot APT data, tightly followed by the ratio measured by ERDA, which proves that the compositional correction can be done based only on data from the APT measurement itself. The accuracy was significantly higher in the homogeneous $\mathrm{ZrB}_{2.0}$ than in the strongly textured $\mathrm{ZrB}_{2.5}$, which is attributed to the heterogeneity in combination with differences 
in probed volume, while the precision was similar. This suggests that further tests on homogeneous materials with well-known compositions are required to better determine the accuracy of the pile-up pairs correction, which is expected to be slightly conservative in most situations. Given accurate isotopic ratios and proper background correction, all compositional correction procedures tested in this study perform well.

A continuous B-rich column boundary network was found in $\mathrm{ZrB}$ 2.5. At the same time, the in-plane electrical resistivity increased $\sim 2.4$ times, suggesting that the grain boundaries are interfering with the conduction electrons.

Grain stoichiometry measurements show up to 4 at. \% deviations from stoichiometry, suggesting that $\mathrm{ZrB}_{2}$ is not a line compound, although it has a very narrow composition range.

\section{Acknowledgements}

The authors would like to acknowledge Jun Lu for TEM micrographs and STEM images, Daniel Primetzhofer for ERDA measurements at the Ångström laboratory in Uppsala, and Babak Bakhit for help with the ERDA data analysis. L. T. acknowledges the Swedish Research Council (VR) through the contract 621-2010-3921 for financial support. H. H. acknowledges the Swedish Government Strategic Research Area in Materials Science on Advanced Functional Materials at Linköping University (Faculty Grant SFO-Mat-LiU No. 2009-00971) for financial support. The Knut and Alice Wallenberg Foundation is acknowledged for the Project Grant (The Boride Frontier, KAW 2015.0043) and a Scholar Grant to L. H. (KAW 2016.0358).

\section{Data availability}

The raw/processed data required to reproduce these findings cannot be shared at this time due to technical or time limitations.

\section{Declarations of interest}

None 


\section{References}

[1] C. Mitterer, Borides in Thin Film Technology, J. Solid State Chem. 133 (1997) 279291. doi:10.1006/jssc.1997.7456.

[2] P.H. Mayrhofer, C. Mitterer, L. Hultman, H. Clemens, Microstructural design of hard coatings, Prog. Mater. Sci. 51 (2006) 1032-1114. doi:10.1016/j.pmatsci.2006.02.002.

[3] Q. Gu, G. Krauss, W. Steurer, Transition Metal Borides: Superhard versus Ultraincompressible, Adv. Mater. 20 (2008) 3620. doi:10.1002/adma.200703025.

[4] H.O. Pierson, Handbook of refractory carbides and nitrides: properties, characteristics, processing and applications, Noyes publications, New Jersey, 1996.

[5] E.K. Storms, The refractory carbides, Academic Press, New York, 1967.

[6] L. Brewer, D.L. Sawyer, D.H. Templeton, C.H. Dauben, A Study of the Refractory Borides, J. Am. Ceram. Soc. 34 (1951) 173-179. doi:10.1111/j.11512916.1951.tb11631.x.

[7] T. Lundström, Transition Metal Borides, in: V.I. Matkovich (Ed.), Boron and Refractory Borides, Springer Berlin Heidelberg, Berlin, Heidelberg, 1977: pp. 351376. doi:10.1007/978-3-642-66620-9_20.

[8] L. Tengdelius, E. Broitman, J. Lu, F. Eriksson, J. Birch, T. Nyberg, L. Hultman, H. Högberg, Hard and elastic epitaxial $\mathrm{ZrB}_{2}$ thin films on $\mathrm{Al}_{2} \mathrm{O}_{3}(0001)$ substrates deposited by magnetron sputtering from a $\mathrm{ZrB}_{2}$ compound target., Acta Mater. 111 (2016) 166-172.

[9] G.-D. Sim, Y.S. Choi, D. Lee, K.H. Oh, J.J. Vlassak, High tensile strength of sputterdeposited $\mathrm{ZrB}_{2}$ ceramic thin films measured up to $1016 \mathrm{~K}$, Acta Mater. 113 (2016) 32-40. doi:10.1016/j.actamat.2016.04.047.

[10] I. Petrov, A. Hall, A.B. Mei, N. Nedfors, I. Zhirkov, J. Rosen, A. Reed, B. Howe, G. Greczynski, J. Birch, L. Hultman, J.E. Greene, Controlling the boron-to-titanium ratio in magnetron-sputter-deposited $\mathrm{TiB}_{\mathrm{x}}$ thin films, J. Vac. Sci. Technol. A. 35 (2017) 050601. doi:10.1116/1.4982649.

[11] B. Bakhit, I. Petrov, J.E. Greene, L. Hultman, J. Rosén, G. Greczynski, Controlling the $\mathrm{B} / \mathrm{Ti}$ ratio of $\mathrm{TiB}_{\mathrm{x}}$ thin films grown by high-power impulse magnetron sputtering, J. Vac. Sci. Technol. A. 36 (2018) 030604. doi:10.1116/1.5026445.

[12] B. Bakhit, D.L.J. Engberg, J. Lu, J. Rosen, H. Högberg, L. Hultman, I. Petrov, J.E. Greene, G. Greczynski, Strategy for simultaneously increasing both hardness and toughness in $\mathrm{ZrB}_{2}$-rich $\mathrm{Zr}_{1-x} \mathrm{Tax}_{\mathrm{B}}$ thin films, J. Vac. Sci. Technol. A. 37 (2019) 031506. doi:10.1116/1.5093170.

[13] B. Post, F.W. Glaser, D. Moskowitz, Transition metal diborides, Acta Metall. 2 (1954) 20-25. doi:10.1016/0001-6160(54)90090-5.

[14] J. Chen, J.A. Barnard, Growth, structure and stress of sputtered $\mathrm{TiB}_{2}$ thin films, Mater. Sci. Eng. A. 191 (1995) 233-238. doi:10.1016/0921-5093(94)09632-7.

[15] M. Berger, E. Coronel, E. Olsson, Microstructure of d.c. magnetron sputtered TiB2 coatings, Surf. Coat. Technol. 185 (2004) 240-244. doi:10.1016/j.surfcoat.2003.12.029.

[16] P.H. Mayrhofer, C. Mitterer, J.G. Wen, J.E. Greene, I. Petrov, Self-organized nanocolumnar structure in superhard $\mathrm{TiB}_{2}$ thin films, Appl. Phys. Lett. 86 (2005) 131909. doi:10.1063/1.1887824.

[17] M. Mikula, B. Grančič, T. Roch, T. Plecenik, I. Vávra, E. Dobročka, A. Šatka, V. Buršíková, M. Držík, M. Zahoran, A. Plecenik, P. Kúš, The influence of low-energy ion 
bombardment on the microstructure development and mechanical properties of $\mathrm{TiB}_{\mathrm{x}}$ coatings, Vacuum. 85 (2011) 866-870. doi:10.1016/j.vacuum.2010.12.011.

[18] F. Lofaj, T. Moskalewicz, G. Cempura, M. Mikula, J. Dusza, A. Czyrska-Filemonowicz, Nanohardness and tribological properties of nc-TiB2 coatings, J. Eur. Ceram. Soc. 33 (2013) 2347-2353. doi:10.1016/j.jeurceramsoc.2013.02.024.

[19] T.B. Massalski, J.L. Murray, L.H. Bennett, H. Baker, eds., Binary alloy phase diagrams, American Society for Metals, Ohio, 1986.

[20] G.S. Collins, Nonstoichiometry in line compounds, J. Mater. Sci. 42 (2007) 19151919. doi:10.1007/s10853-006-0055-2.

[21] N. Nedfors, O. Tengstrand, J. Lu, P. Eklund, P.O.Å. Persson, L. Hultman, U. Jansson, Superhard $\mathrm{NbB}_{2-\mathrm{x}}$ thin films deposited by dc magnetron sputtering, Surf. Coat. Technol. 257 (2014) 295-300. doi:10.1016/j.surfcoat.2014.07.087.

[22] K.L. Dahm, L.R. Jordan, J. Haase, P.A. Dearnley, Magnetron sputter deposition of chromium diboride coatings, Surf. Coat. Technol. 108-109 (1998) 413-418. doi:10.1016/S0257-8972(98)00568-4.

[23] S. Zhang, Z. Wang, P. Guo, P. Ke, M. Odén, A. Wang, Temperature induced superhard $\mathrm{CrB} 2$ coatings with preferred (001) orientation deposited by DC magnetron sputtering technique, Surf. Coat. Technol. 322 (2017) 134-140. doi:10.1016/j.surfcoat.2017.05.030.

[24] A.A. Goncharov, V.A. Konovalov, S.N. Dub, V.A. Stupak, V.V. Petukhov, Structure, composition, and physicomechanical characteristics of tantalum diboride films, Phys. Met. Metall. 107 (2009) 285-290. doi:10.1134/S0031918X09030107.

[25] F. Ge, C. Chen, R. Shu, F. Meng, P. Li, F. Huang, Hard and wear resistant VB2 coatings deposited by pulsed DC magnetron sputtering, Vacuum. 135 (2017) 66-72. doi:10.1016/j.vacuum.2016.09.014.

[26] A. O’Brien, B. Villegas, J.Y. Gu, Sputtered magnesium diboride thin films: Growth conditions and surface morphology, Physica C. 469 (2009) 39-43. doi:10.1016/j.physc.2008.10.006.

[27] C. Buzea, T. Yamashita, Review of superconducting properties of $\mathrm{MgB}_{2}$, Supercond. Sci. Technol. 14 (2001) R115-R146.

[28] V.A. Gasparov, N.S. Sidorov, I.I. Zver'kova, M.P. Kulakov, Electron transport in diborides: Observation of superconductivity in $\mathrm{ZrB}_{2}$, JETP Lett. 73 (2001) 532-535. doi:10.1134/1.1387521.

[29] N. Barbero, T. Shiroka, B. Delley, T. Grant, A.J.S. Machado, Z. Fisk, H.-R. Ott, J. Mesot, Doping-induced superconductivity of $\mathrm{ZrB}_{2}$ and $\mathrm{HfB}_{2}$, Phys. Rev. B. 95 (2017). doi:10.1103/PhysRevB.95.094505.

[30] M.-A. Nicolet, Diffusion barriers in thin films, Thin Solid Films. 52 (1978) 415-443. doi:10.1016/0040-6090(78)90184-0.

[31] H. Kinoshita, S. Otani, S. Kamiyama, H. Amano, I. Akasaki, J. Suda, H. Matsunami, Zirconium Diboride (0001) as an Electrically Conductive Lattice-Matched Substrate for Gallium Nitride, Jpn. J. Appl. Phys. Part 2. 40 (2001) L1280-L1282. doi:10.1143/JJAP.40.L1280.

[32] J. Tolle, R. Roucka, I.S.T. Tsong, C. Ritter, P.A. Crozier, A.V.G. Chizmeshya, J. Kouvetakis, Epitaxial growth of group III nitrides on silicon substrates via a reflective lattice-matched zirconium diboride buffer layer, Appl. Phys. Lett. 82 (2003) 2398-2400. doi:10.1063/1.1566099.

[33] Y. Yamada-Takamura, Z.T. Wang, Y. Fujikawa, T. Sakurai, Q.K. Xue, J. Tolle, P.-L. Liu, A.V.G. Chizmeshya, J. Kouvetakis, I.S.T. Tsong, Surface and Interface Studies of GaN 
Epitaxy on Si(111) via ZrB 2 Buffer Layers, Phys. Rev. Lett. 95 (2005).

doi:10.1103/PhysRevLett.95.266105.

[34] E.A. Serban, J. Palisaitis, M. Junaid, L. Tengdelius, H. Högberg, L. Hultman, P. Persson, J. Birch, C.-L. Hsiao, Magnetron Sputter Epitaxy of High-Quality GaN Nanorods on Functional and Cost-Effective Templates/Substrates, Energies. 10 (2017) 1322. doi:10.3390/en10091322.

[35] G. Da Costa, H. Wang, S. Duguay, A. Bostel, D. Blavette, B. Deconihout, Advance in multi-hit detection and quantization in atom probe tomography, Rev. Sci. Instrum. 83 (2012) 123709.

[36] F. Meisenkothen, T.J. Prosa, E.B. Steel, R. Prakash Kolli, The Role of Multi-Hit Detection Events on the Accurate Measurement of Boron in Atom Probe Tomography, Microsc. Microanal. 20 (2014) 1962-1963. doi:10.1017/S1431927614011544.

[37] F. Meisenkothen, E.B. Steel, T.J. Prosa, K.T. Henry, P.R. Kolli, Effects of detector deadtime on quantitative analyses involving boron and multi-hit detection events in atom probe tomography, Ultramicroscopy. 159 (2015) 101-111. doi:10.1016/j.ultramic.2015.07.009.

[38] A. Szczepaniak, H. Springer, R. Aparicio-Fernández, C. Baron, D. Raabe, Strengthening $\mathrm{Fe}-\mathrm{TiB}_{2}$ based high modulus steels by precipitations, Mater. Des. 124 (2017) 183-193. doi:10.1016/j.matdes.2017.03.042.

[39] K. Wedderhoff, C.A. Kleint, A. Shariq, S. Teichert, A study of nanoscale TiB2 precipitation during titanium silicidation using atom probe tomography, Thin Solid Films. 519 (2011) 7826-7829. doi:10.1016/j.tsf.2011.05.068.

[40] J. Deges, R. Rablbauer, G. Frommeyer, A. Schneider, Observation of boron enrichments in a heat-treated quasi-binary hypoeutectic NiAl-HfB 2 alloy by means of atom probe field-ion microscopy (APFIM), Surf. Interface Anal. 39 (2007) 251256. doi:10.1002/sia.2524.

[41] M. Magnuson, L. Tengdelius, G. Greczynski, L. Hultman, H. Högberg, Chemical bonding in epitaxial ZrB2 studied by X-ray spectroscopy, Thin Solid Films. 649 (2018) 89-96. doi:10.1016/j.tsf.2018.01.021.

[42] M. Dahlqvist, U. Jansson, J. Rosen, Influence of boron vacancies on phase stability, bonding and structure of $M B_{2}(M=\mathrm{Ti}, \mathrm{Zr}, \mathrm{Hf}, \mathrm{V}, \mathrm{Nb}, \mathrm{Ta}, \mathrm{Cr}, \mathrm{Mo}, \mathrm{W})$ with $\mathrm{AlB}_{2}$ type structure, J. Phys.: Condens. Matter. 27 (2015) 435702. doi:10.1088/09538984/27/43/435702.

[43] J. Neidhardt, S. Mráz, J.M. Schneider, E. Strub, W. Bohne, B. Liedke, W. Möller, C. Mitterer, Experiment and simulation of the compositional evolution of Ti-B thin films deposited by sputtering of a compound target, J. Appl. Phys. 104 (2008) 063304. doi:10.1063/1.2978211.

[44] L. Tengdelius, J. Birch, J. Lu, L. Hultman, U. Forsberg, E. Janzén, H. Högberg, Magnetron sputtering of epitaxial $\mathrm{ZrB}_{2}$ thin films on $4 \mathrm{H}-\mathrm{SiC}(0001)$ and $\mathrm{Si}(111)$, Phys. Status Solidi A. 211 (2014) 636-640. doi:10.1002/pssa.201330308.

[45] L. Tengdelius, G. Greczynski, M. Chubarov, J. Lu, U. Forsberg, L. Hultman, E. Janzén, H. Högberg, Stoichiometric, epitaxial $\mathrm{ZrB}_{2}$ thin films with low oxygen-content deposited by magnetron sputtering from a compound target: Effects of deposition temperature and sputtering power, J. Cryst. Growth. 430 (2015) 55-62.

[46] M.S. Janson, CONTES Conversion of Time-Energy Spectra - a Program for ERDA Data Analysis, Uppsala University, 2004.

[47] K. Arstila, J. Julin, M.I. Laitinen, J. Aalto, T. Konu, S. Kärkkäinen, S. Rahkonen, M. Raunio, J. Itkonen, J.-P. Santanen, T. Tuovinen, T. Sajavaara, Potku - New analysis 
software for heavy ion elastic recoil detection analysis, Nucl. Instrum. Methods Phys. Res., Sect. B. 331 (2014) 34-41. doi:10.1016/j.nimb.2014.02.016.

[48] K. Thompson, D. Lawrence, D.J. Larson, J.D. Olson, T.F. Kelly, B. Gorman, In situ sitespecific specimen preparation for atom probe tomography, Ultramicroscopy. 107 (2007) 131-139. doi:10.1016/j.ultramic.2006.06.008.

[49] K. Thompson, B. Gorman, D. Larson, B. van Leer, L. Hong, Minimization of Ga Induced FIB Damage Using Low Energy Clean-up, Microsc. Microanal. 12 (2006) 1736-1737. doi:10.1017/S1431927606065457.

[50] B.P. Geiser, D.J. Larson, E. Oltman, S. Gerstl, D. Reinhard, T.F. Kelly, T.J. Prosa, Widefield-of-view atom probe reconstruction, Microsc. Microanal. 15 (2009) 292-293. doi:10.1017/S1431927609098249.

[51] JCPDS - International Centre for Diffraction Data, Zirconium Diboride ( $\left.\mathrm{ZrB}_{2}\right)$ Ref. ID 00-034-0423, (n.d.).

[52] B.P. Geiser, T.F. Kelly, D.J. Larson, J. Schneir, J.P. Roberts, Spatial Distribution Maps for Atom Probe Tomography, Microsc. Microanal. 13 (2007) 437-447. doi:10.1017/S1431927607070948.

[53] F. De Geuser, B. Gault, A. Bostel, F. Vurpillot, Correlated field evaporation as seen by atom probe tomography, Surf. Sci. 601 (2007) 536-543. doi:10.1016/j.susc.2006.10.019.

[54] D.L.J. Engberg, L.J.S. Johnson, J. Jensen, M. Thuvander, L. Hultman, Resolving mass spectral overlaps in atom probe tomography by isotopic substitutions - case of TiSi15N, Ultramicroscopy. 184 (2018) 51-60. doi:10.1016/j.ultramic.2017.08.004.

[55] F. Tang, B. Gault, S.P. Ringer, J.M. Cairney, Optimization of pulsed laser atom probe (PLAP) for the analysis of nanocomposite Ti-Si-N films, Ultramicroscopy. 110 (2010) 836-843. doi:10.1016/j.ultramic.2010.03.003.

[56] L.J.S. Johnson, M. Odén, L. Hultman, M. Thuvander, K. Stiller, Spinodal decomposition of $\mathrm{Ti}_{0.33} \mathrm{Al}_{0.67} \mathrm{~N}$ thin films studied by atom probe tomography, Thin Solid Films. 520 (2012) 4362-4368. doi:10.1016/j.tsf.2012.02.085.

[57] I. Povstugar, P.-P. Choi, D. Tytko, J.-P. Ahn, D. Raabe, Interface-directed spinodal decomposition in TiAlN/CrN multilayer hard coatings studied by atom probe tomography, Acta Mater. 61 (2013) 7534-7542. doi:10.1016/j.actamat.2013.08.028.

[58] L.J.S. Johnson, N. Ghafoor, D. Engberg, M. Thuvander, K. Stiller, M. Odén, L. Hultman, Self-organized nanostructuring in $\mathrm{Zr}_{0.69} \mathrm{Al}_{0.31} \mathrm{~N}$ thin films studied by atom probe tomography, Thin Solid Films. 615 (2016) 233-238. doi:10.1016/j.tsf.2016.07.034.

[59] M. Hans, J.M. Schneider, On the chemical composition of TiAlN thin films Comparison of ion beam analysis and laser-assisted atom probe tomography with varying laser pulse energy, Thin Solid Films. (2019). doi:10.1016/j.tsf.2019.04.026.

[60] M. Thuvander, J. Weidow, J. Angseryd, L.K.L. Falk, F. Liu, M. Sonestedt, K. Stiller, H.0 . Andrén, Quantitative atom probe analysis of carbides, Ultramicroscopy. 111 (2011) 604-608. doi:10.1016/j.ultramic.2010.12.024.

[61] Z. Peng, P.-P. Choi, B. Gault, D. Raabe, Evaluation of Analysis Conditions for LaserPulsed Atom Probe Tomography: Example of Cemented Tungsten Carbide, Microsc. Microanal. 23 (2017) 431-442. doi:10.1017/S1431927616012654.

[62] J. Angseryd, F. Liu, H.-O. Andrén, M. Thuvander, S.S.A. Gerstl, Quantitative APT analysis of Ti(C,N), Ultramicroscopy. 111 (2011) 609-614. doi:10.1016/j.ultramic.2011.01.031. 
[63] T. Boll, M. Thuvander, S. Koch, J.N. Wagner, N. Nedfors, U. Jansson, K. Stiller, An APT investigation of an amorphous Cr-B-C thin film, Ultramicroscopy. 159 (2015) 217222. doi:10.1016/j.ultramic.2015.01.001.

[64] D.W. Saxey, Correlated ion analysis and the interpretation of atom probe mass spectra, Ultramicroscopy. 111 (2011) 473-479. doi:10.1016/j.ultramic.2010.11.021.

[65] B. Deconihout, F. Vurpillot, M. Bouet, L. Renaud, Improved ion detection efficiency of microchannel plate detectors, Rev. Sci. Instrum. 73 (2002) 1734-1740. doi:10.1063/1.1461882.

[66] K. Kruska, D.K. Schreiber, Background Recovery through the Quantification of Delayed Evaporation Multi-Ion Events in Atom-Probe Data, Microsc. Microanal. 21 (2015) 857-858. doi:10.1017/S1431927615005085.

[67] S. Pedrazzini, A.J. London, B. Gault, D. Saxey, S. Speller, C.R.M. Grovenor, M. Danaie, M.P. Moody, P.D. Edmondson, P.A.J. Bagot, Nanoscale Stoichiometric Analysis of a High-Temperature Superconductor by Atom Probe Tomography, Microsc. Microanal. 23 (2017) 414-424. doi:10.1017/S1431927616012757.

[68] Z. Peng, F. Vurpillot, P.-P. Choi, Y. Li, D. Raabe, B. Gault, On the detection of multiple events in atom probe tomography, Ultramicroscopy. 189 (2018) 54-60. doi:10.1016/j.ultramic.2018.03.018.

[69] D.J. Larson, T.J. Prosa, R.M. Ulfig, B.P. Geiser, T.F. Kelly, Local Electrode Atom Probe Microscopy: A User's Guide, Springer, New York, 2013. doi:10.1007/978-1-46148721-0.

[70] D.J. Larson, T.J. Prosa, E. Oltman, D.A. Reinhard, B.P. Geiser, R.M. Ulfig, A. Merkulov, A High Multiple Hits Correction Factor for Atom Probe Tomography, Microsc. Microanal. 24 (2018) 1084-1085. doi:10.1017/S1431927618005901.

[71] F. Liu, H.-O. Andrén, Effects of laser pulsing on analysis of steels by atom probe tomography, Ultramicroscopy. 111 (2011) 633-641. doi:10.1016/j.ultramic.2010.12.012.

[72] I. Fedorova, F.B. Grumsen, J. Hald, H.-O. Andrén, F. Liu, Analyzing Boron in 9-12\% Chromium Steels Using Atom Probe Tomography, Microsc. Microanal. (2019) 1-8. doi:10.1017/S1431927618015726.

[73] U. Rolander, H.-O. Andrén, Statistical Correction for Pile-up in the Atom-Probe Detector System, J. Phys. Colloq. 50 (1989) C8-529-C8-534. doi:10.1051/jphyscol:1989891.

[74] B. Gault, M.P. Moody, J.M. Cairney, S.P. Ringer, Atom probe crystallography, Mater. Today. 15 (2012) 378-386. doi:10.1016/S1369-7021(12)70164-5.

[75] T. Stephan, P.R. Heck, D. Isheim, J.B. Lewis, Correction of dead time effects in laserinduced desorption time-of-flight mass spectrometry: Applications in atom probe tomography, Int. J. Mass Spectrom. 379 (2015) 46-51. doi:10.1016/j.ijms.2014.12.006.

[76] L. Xu, Y. Xiao, A. van Sandwijk, Q. Xu, Y. Yang, Separation of Zirconium and Hafnium: A Review, in: Energy Materials 2014, John Wiley \& Sons, Inc., Hoboken, NJ, USA, 2015: pp. 451-457. doi:10.1002/9781119027973.ch53.

[77] W.G. Fahrenholtz, G.E. Hilmas, I.G. Talmy, J.A. Zaykoski, Refractory Diborides of Zirconium and Hafnium, J. Am. Ceram. Soc. 90 (2007) 1347-1364. doi:10.1111/j.1551-2916.2007.01583.x.

[78] L. Tengdelius, M. Samuelsson, J. Jensen, J. Lu, L. Hultman, U. Forsberg, E. Janzén, H. Högberg, Direct current magnetron sputtered $\mathrm{ZrB}_{2}$ thin films on $4 \mathrm{H}-\mathrm{SiC}(0001)$ and Si(100), Thin Solid Films. 550 (2014) 285-290. doi:10.1016/j.tsf.2013.11.040. 
[79] F. Danoix, G. Grancher, A. Bostel, D. Blavette, Standard deviations of composition measurements in atom probe analyses-Part II: 3D atom probe, Ultramicroscopy. 107 (2007) 739-743. doi:10.1016/j.ultramic.2007.02.005.

[80] J.R. Shappirio, J.J. Finnegan, Synthesis and properties of some refractory transition metal diboride thin films, Thin Solid Films. 107 (1983) 81-87. doi:10.1016/00406090(83)90010-X.

[81] M. Guziewicz, A. Piotrowska, E. Kamińska, K. Gołaszewska, A. Turos, E. Mizera, A. Winiarski, J. Szade, Characteristics of sputter-deposited $\mathrm{TiN}, \mathrm{ZrB}_{2}$ and $\mathrm{W}_{2} \mathrm{~B}$ diffusion barriers for advanced metallizations to GaAs, Solid State Electron. 43 (1999) 10551061. doi:10.1016/S0038-1101(99)00024-6.

[82] M. Samuelsson, J. Jensen, U. Helmersson, L. Hultman, H. Högberg, ZrB2 thin films grown by high power impulse magnetron sputtering from a compound target, Thin Solid Films. 526 (2012) 163-167. doi:10.1016/j.tsf.2012.11.006.

[83] U.K. Chakrabarti, H. Barz, W.C. Dautremont-Smith, J.W. Lee, T.Y. Kometani, Deposition of zirconium boride thin films by direct current triode sputtering, J. Vac. Sci. Technol. A. 5 (1987) 196-201. doi:10.1116/1.574103.

[84] P. de la Mora, M. Castro, G. Tavizon, Comparative study of the electronic structure of alkaline-earth borides $\left(\mathrm{MeB}_{2} ; \mathrm{Me}=\mathrm{Mg}, \mathrm{Al}, \mathrm{Zr}, \mathrm{Nb}\right.$, and $\left.\mathrm{Ta}\right)$ and their normal-state conductivity, J. Solid State Chem. 169 (2002) 168-175. doi:10.1016/S00224596(02)00045-2.

[85] X. Zhang, X. Luo, J. Han, J. Li, W. Han, Electronic structure, elasticity and hardness of diborides of zirconium and hafnium: First principles calculations, Comput. Mater. Sci. 44 (2008) 411-421. doi:10.1016/j.commatsci.2008.04.002.

[86] M.K. Miller, M.G. Hetherington, Local magnification effects in the atom probe, Surf. Sci. 246 (1991) 442-449. doi:10.1016/0039-6028(91)90449-3.

[87] F. Vurpillot, A. Bostel, D. Blavette, Trajectory overlaps and local magnification in three-dimensional atom probe, Appl. Phys. Lett. 76 (2000) 3127-3129. doi:10.1063/1.126545.

[88] B. Gault, M.P. Moody, J.M. Cairney, S.P. Ringer, Atom Probe Microscopy, Springer, New York, 2012. doi:10.1007/978-1-4614-3436-8.

[89] P.J. Felfer, B. Gault, G. Sha, L. Stephenson, S.P. Ringer, J.M. Cairney, A New Approach to the Determination of Concentration Profiles in Atom Probe Tomography, Microsc. Microanal. 18 (2012) 359-364. doi:10.1017/S1431927611012530.

[90] B. Gault, F. Danoix, K. Hoummada, D. Mangelinck, H. Leitner, Impact of directional walk on atom probe microanalysis, Ultramicroscopy. 113 (2012) 182-191. doi:10.1016/j.ultramic.2011.06.005.

[91] R.M. Ulfig, D.J. Larson, T.F. Kelly, P.H. Clifton, T.J. Prosa, D.R. Lenz, E.X. Oltman, Performance Advances in LEAP systems, Microsc. Microanal. 20 (2014) 1120-1121. doi:10.1017/S1431927614007338.

[92] T.L. Martin, A.J. London, B. Jenkins, S.E. Hopkin, J.O. Douglas, P.D. Styman, P.A.J. Bagot, M.P. Moody, Comparing the Consistency of Atom Probe Tomography Measurements of Small-Scale Segregation and Clustering Between the LEAP 3000 and LEAP 5000 Instruments, Microsc. Microanal. 23 (2017) 227-237. doi:10.1017/S1431927617000356.

[93] M. Thuvander, A. Kvist, L.J.S. Johnson, J. Weidow, H.-O. Andrén, Reduction of multiple hits in atom probe tomography, Ultramicroscopy. 132 (2013) 81-85. doi:10.1016/j.ultramic.2012.12.005.

[94] A. Menand, Isotopic variations in field evaporation charge-state of boron ions, J. Phys. D: Appl. Phys. 17 (1984) 203. doi:10.1088/0022-3727/17/1/026. 
[95] D. Blavette, P. Duval, L. Letellier, M. Guttmann, Atomic-scale APFIM and TEM investigation of grain boundary microchemistry in Astroloy nickel base superalloys, Acta Mater. 44 (1996) 4995-5005. doi:10.1016/S1359-6454(96)00087-0. 